\title{
Function Computation through a Bidirectional Relay
}

\author{
Jithin Ravi and Bikash Kumar Dey
}

\begin{abstract}
We consider a function computation problem in a three node wireless network. Nodes A and B observe two correlated sources $X$ and $Y$ respectively, and want to compute a function $f(X, Y)$. To achieve this, nodes A and B send messages to a relay node $\mathrm{C}$ at rates $R_{A}$ and $R_{B}$ respectively. The relay $\mathrm{C}$ then broadcasts a message to $\mathrm{A}$ and $\mathrm{B}$ at rate $R_{C}$. We allow block coding, and study the achievable region of rate triples under both zero-error and $\epsilon$-error. As a preparation, we first consider a broadcast network from the relay to $\mathrm{A}$ and $\mathrm{B}$. $\mathrm{A}$ and $\mathrm{B}$ have side information $X$ and $Y$ respectively. The relay node $\mathrm{C}$ observes both $X$ and $Y$ and broadcasts an encoded message to $\mathrm{A}$ and $\mathrm{B}$. We want to obtain the optimal broadcast rate such that $\mathrm{A}$ and $\mathrm{B}$ can recover the function $f(X, Y)$ from the received message and their individual side information $X$ and $Y$ respectively. For this problem, we show equivalence between $\epsilon$-error and zero-error computations- this gives a rate characterization for zero-error computation. As a corollary, this also gives a rate characterization for the relay network under zero-error for a class of functions called component-wise one-to-one functions when the support set of $p_{X Y}$ is full. For the relay network, the zero-error rate region for arbitrary functions is characterized in terms of graph coloring of some suitably defined probabilistic graphs. We then give a single-letter inner bound to this rate region. Further, we extend the graph theoretic ideas to address the $\epsilon$-error problem and obtain a single-letter inner bound.
\end{abstract}

\section{Index Terms}

Distributed source coding, function computation, zero-error information theory.

\section{INTRODUCTION}

Distributed computation of distributed data over a network has been investigated in various flavours for a long time. Gathering all the data at the nodes where a function needs to be computed is wasteful in most situations. So intermediate nodes also help by doing some processing of the data to reduce the communication load on the links. Such computation frameworks are known as distributed function computation or in-network function computation [1]-[6].

This paper was presented in part at the IEEE Information Theory Workshop (ITW), Jerusalem, Israel, April 2015 and at the IEEE GLOBECOM NetCod 2016, Washington, DC, USA, December 2016. This work was supported by the Department of Science and Technology under grant SB/S3/EECE/057/2013 and by Information Technology Research Academy under grant ITRA/15(64)/Mobile/USEAADWN/01.

J. Ravi and B. K. Dey are with the Department of Electrical Engineering at IIT Bombay, Mumbai, INDIA-400076. Email: $\{$ rjithin,bikash\}@ee.iitb.ac.in. 


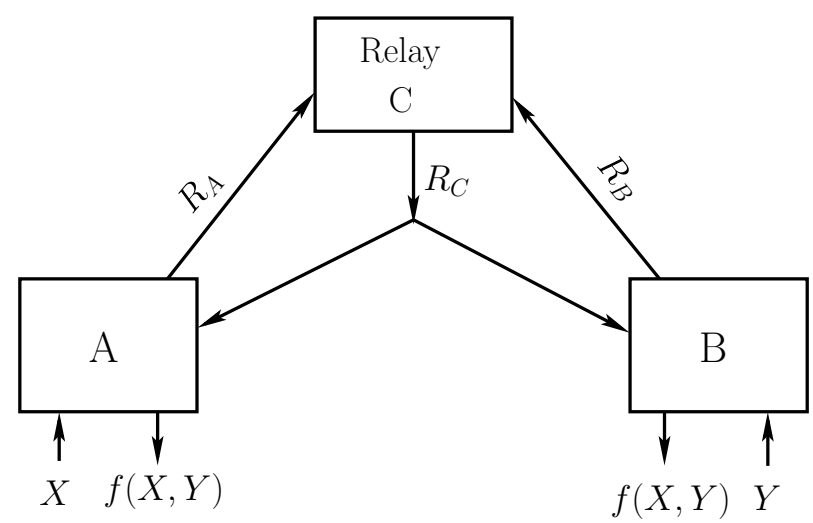

Fig. 1: Function computation in bidirectional relay network (RN)

We consider the problem of function computation in a wireless relay network (RN) with three nodes as shown in Fig. 1. Nodes A and B have two correlated random variables $X$ and $Y$ respectively. They have infinite i.i.d. realizations of these random variables. They can communicate directly to a relay node $\mathrm{C}$ over independent errorfree links. The relay node $\mathrm{C}$ can send a message to both A and B over a noise-less broadcast link. Nodes A and B want to compute a function $f(X, Y)=Z$. We allow block coding of arbitrarily large block length $n$. We allow two phases of communication. In the first phase, both $\mathrm{A}$ and $\mathrm{B}$ send individual messages to $\mathrm{C}$ at rates $R_{A}$ and $R_{B}$ over the respective independent links. In the second phase, the relay broadcasts a message to $\mathrm{A}$ and $\mathrm{B}$ at rate $R_{C}$.

The broadcasting relay in the model captures one aspect of wireless networks. We consider our function computation problem over this network under zero-error and $\epsilon$-error criteria. Under zero-error, both nodes want to compute the function with no error. Under $\epsilon$-error, the probability of error in computing the function should go to zero as block length tends to infinity. A special case of this problem have been studied in [7], [8]. Exchanging $X$ and $Y$ was considered in [7], and the rate region was characterized in the $\epsilon$-error setting. For this problem, some single-letter inner and outer bounds were given for the rate-distortion function in [8].

As a preparation to address the problem in Fig. 1. we first consider the broadcast function network with complementary side information (BFN-CSI) shown in Fig. 2. This problem arises as a special case of the function computation problem in the relay network, when $\mathrm{A}$ and $\mathrm{B}$ communicate $X$ and $Y$ to the relay node. In the relay network, rate $R_{C}$ attains its minimum when the relay has $X$ and $Y$. So the optimal broadcast rate for the problem in Fig. 2 is the minimum possible rate $R_{C}$ in the relay network. For the broadcast function network, the optimal $\epsilon$-error rate can be shown to be $\max \{H(Z \mid Y), H(Z \mid Y)\}$ using the Slepian-Wolf result. We study this problem under zero-error criteria.

The problem of zero-error source coding with receiver side information was first studied for fixed length coding by Witsenhausen in [9] using a "confusability graph" $G_{X \mid Y}$. The minimum rate was characterized in terms of the chromatic number of its AND product graphs $G_{X \mid Y}^{\wedge n}$. The same problem was later considered in [10] under variable 


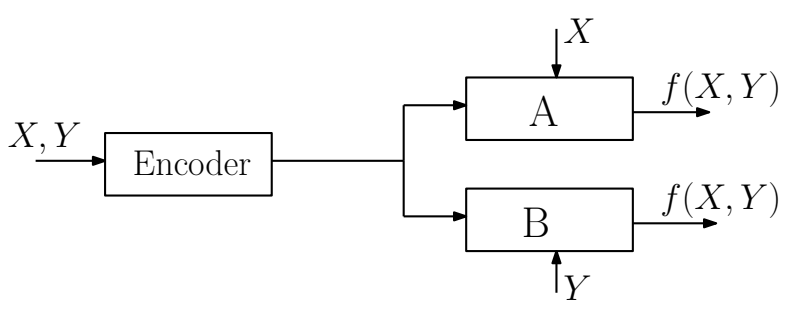

Fig. 2: Broadcast function network with complementary side information (BFN-CSI)

length coding, and the minimum rate was shown to be the limit of the normalized chromatic entropy of $G_{X \mid Y}^{\wedge n}$. This asymptotic rate was later shown [11] to be the complementary graph entropy [12] of $G_{X \mid Y}$. However, A single-letter characterization for complementary graph entropy is still unknown.

In the absence of a single-letter characterization of zero-error source coding problems, many authors have studied their problems under a stricter decoding requirement, known as the "unrestricted input" setup [10], [14], [15]. In this setup, even for a source vector which has some zero-probability components (and thus the vector itself having probability 0), the decoder is required to reproduce the desired symbols for the other components of the vector. Unrestricted input setup was introduced in [10], and for the problem addressed in [10], the optimum rate under unrestricted input setup was shown to be the graph entropy of the confusability graph which has a single letter characterization. On one hand, under unrestricted input setup, computation problems are sometimes tractable when the original zero-error computation problems are not. On the other hand, protocols for the unrestricted input setup are clearly also valid protocols for the original zero-error decoding problem. So achievable rates under unrestricted input setup give upper bounds on the optimal zero-error rates. Shayevitz [15] also studied the unrestricted input version of their problem. In all these models, the unrestricted input setup is represented by the OR product of a suitable confusability graph. In contrast, for our function computation problem in the relay network, the unrestricted input setup is not represented by the OR products of the confusability graph.

For distributed coding of two sources and joint decoding, a single-letter characterization was given for the unrestricted input version in [14]. Most related recent work to our present work is [15], where a decoder having side information $Z$ wants to compute a function $f(X, Y, Z)$ using a message encoded by a relay, which in turn receives two messages encoded by two sources $X$ and $Y$. Single-letter inner and outer bounds were given for the unrestricted input setup.

The problem of broadcast with side information, has been studied extensively in the literature (see [16]- [21] and references therein). Index coding (see [22]- [26]) is a special case of broadcast with side information, and it is related to our work. In index coding, a server has access to $K$ binary independent and uniformly distributed random variables and the receivers have access to different subsets of these messages. Each receiver wants to recover an arbitrary subset of the messages using its side information and the message broadcasted by the server. The goal is 
to minimize the broadcast rate of the message sent by the server. A computable characterization of the optimum broadcast rate for the general index coding problem is still unknown. For our broadcast function network (Fig. 2), instead of recovering the messages, we consider the problem of computing a function of the messages. For this problem, the optimal $\epsilon$-error rate is $\max \{H(Z \mid X), H(Z \mid Y)\}$ (using Slepian-Wolf result), thus it is a lower bound for the optimal zero-error rate. We show that the rate $\max \{H(Z \mid X), H(Z \mid Y)\}$ is achievable under zero-error.

For the relay network, we study the function computation problem under zero-error. Suitable graphs are defined to address the problem. We first consider computing a component-wise one-to-one function at both the end nodes. Note that computing a component-wise one-to-one function in the relay network is the equivalent to exchanging $X$ and $Y$ through the relay. Building on our results on the broadcast function network, we give a single-letter characterization of the rate region for computing a component-wise one-to-one function when the support set of $p_{X Y}$ is full. For arbitrary functions, we study the problem under unrestricted input setup and provide a multiletter characterization of the rate region. Then we provide a single-letter inner bound for this region, which is also an inner bound for the zero-error problem.

Next, we consider the function computation problem in the relay network under $\epsilon$-error. For this problem, we use the graph theoretic ideas developed for zero-error, to get a single-letter inner bound for the rate region.

\section{A. Contributions and organization of the paper}

We list the contributions of this paper below.

- For the zero-error function computation problem shown in Fig. 2, in Theorem 1 , we show that the optimal zero-error broadcast rate is same as optimal $\epsilon$-error rate which has a single-letter characterization. Using this result, we give a single-letter characterization of the rate region for computing a component-wise one-to-one function in the relay network (Fig. 1) when the support set of $p_{X Y}$ is full. We then argue that when $X$ and $Y$ are independent, exchanging $(X, Y)$ in the relay network has the same rate region under zero-error and $\epsilon$-error.

- We consider the zero-error function computation problem in the relay network (Fig. 1) under the unrestricted input setup. This setup is a more constrained version of the zero-error problem. We give a multiletter characterization of the rate region under this setup as well as for the zero-error problem (Theorem 2). The multiletter characterization is obtained using coloring of some suitably defined graphs. Our arguments based on coloring are similar to [15]. We show that if $p_{X Y}$ has full support, then the relay can also compute the function if $\mathrm{A}$ and B can compute it with zero-error (Theorem 4).

- For the unrestricted input setup, we propose two achievable schemes whose time sharing gives a single-letter inner bound for the corresponding rate region (Theorem 3 ). 
- The function computation problem in Fig. 1 is then addressed under $\epsilon$-error. We extend the graph theoretic ideas used for zero-error computation to $\epsilon$-error computation. Similar to the two achievable schemes for zero-error computation, we give an inner bound for the rate region using two achievable schemes for $\epsilon$-error computation (Theorem 5). The cutset outer bound is given in Lemma 1

- For two functions $f_{1}, f_{2}$ of $(X, Y)$, we give a graph theoretic sufficient condition under which the rate region for computing $f_{1}$ is a subset of the rate region for computing $f_{2}$. This condition holds for both zero-error and $\epsilon$-error computations (Theorem 6). Using this result, we give a class of functions for which the rate region is the same as the region for exchanging $(X, Y)$.

The organization of the paper is as follows. Problem formulations for zero-error and $\epsilon$-error are given in Section $\mathrm{II}-\mathrm{A}$ and in Section $\mathrm{II}-\mathrm{B}$ respectively. Some graph theoretic definitions are given in Section II-C. We provide our results for zero-error computation in Section III-A. The $\epsilon$-error results are given in Section III-B. The proof of the results for zero-error computation and $\epsilon$-error computation are given in Section $[\mathrm{IV}$ and Section $\mathrm{V}$ respectively. We conclude our paper in Section VI.

\begin{tabular}{|c|c|c|}
\hline & Zero-error & $\epsilon$-error \\
\hline $\begin{array}{l}\text { BFN- } \\
\text { CSI }\end{array}$ & - Complete characterization (Theorem 1 ) & $\begin{array}{l}\text { - Follows directly from Slepian-Wolf } \\
\text { results }\end{array}$ \\
\hline \multirow[t]{2}{*}{ RN } & $\begin{array}{l}\text { - Multiletter characterization (Theorem } 2 \\
\text { - Single-letter characterization for CWOOF when } \\
\text { support set is full (Corollary } 1 \text { ) } \\
\text { - Single-letter inner bound for unrestricted inputs } \\
\text { (Theorem } 3 \\
\text { - A sufficient condition on } p_{X Y} \text { under which the } \\
\text { relay can compute the function in any zero-error } \\
\text { scheme (Theorem 4) }\end{array}$ & $\begin{array}{l}\text { - Cutset outer bound (Lemma } 1 \\
\text { - Single-letter inner bound (Theo- } \\
\text { rem } 5 \text { p }\end{array}$ \\
\hline & - Graph-based sufficient condition for "rate region & for $f_{1} \supseteq$ rate region for $f_{2}$ " (Theorem 6 ) \\
\hline
\end{tabular}

TABLE I: Summary of our results

\section{PROBLEM FORMULATION AND PRELIMINARIES}

Nodes $\mathrm{A}$ and $\mathrm{B}$ observe $X$ and $Y$ respectively from finite alphabet sets $\mathcal{X}$ and $\mathcal{Y}$. Let function $Z=f(X, Y)$ take values in a finite alphabet set $\mathcal{Z} .(X, Y)$ have a joint distribution $p_{X Y}(x, y)$, and their different realizations are i.i.d. In other words, $n$ consecutive realizations $\left(X^{n}, Y^{n}\right)$ are distributed as $\operatorname{Pr}\left(x^{n}, y^{n}\right)=\prod_{i=1}^{n} p_{X Y}\left(x_{i}, y_{i}\right)$ for all $x^{n}=\left(x_{1}, x_{2}, \cdots, x_{n}\right)$ and $y^{n}=\left(y_{1}, y_{2}, \cdots, y_{n}\right)$.

The support set of $(X, Y)$ is defined as $S_{X Y}=\left\{(x, y): p_{X Y}(x, y)>0\right\}$. We use the notion of robust typicality [3] in the following. For $x^{n} \in \mathcal{X}^{n}$, let us denote the number of occurrences of $x \in \mathcal{X}$ in $x^{n}$ by $N\left(x \mid x^{n}\right)$. The set 
of sequences $x^{n} \in \mathcal{X}^{n}$ satisfies

$$
\left|\frac{1}{n} N\left(x \mid x^{n}\right)-p(x)\right| \leq \epsilon \cdot p(x)
$$

for $\epsilon>0$, is called $\epsilon$-robustly typical sequences and is denoted by $T_{\epsilon}^{n}(X)$.

Definition 1 A function $f(x, y)$ is called component-wise one-to-one function (CWOOF) if it satisfies the following:

1) $f(x, y) \neq f\left(x, y^{\prime}\right)$ for all $x \in \mathcal{X}, y, y^{\prime} \in \mathcal{Y}, y \neq y^{\prime}$,

and

2) $f(x, y) \neq f\left(x^{\prime}, y\right)$ for all $y \in \mathcal{Y}, x, x^{\prime} \in \mathcal{X}, x \neq x^{\prime}$.

This class of functions includes the binary XOR function, and in general, the function $a+b \bmod \max (x, y)$, where $x$ and $y$ are positive integers and $0 \leq a \leq x-1,0 \leq b \leq y-1$. Note that computing a component-wise one-to-one function either in the broadcast network or in the relay network is equivalent to recovering both $X$ and $Y$ at nodes $A$ and $B$.

\section{A. Zero-error function computation}

Relay Network: On observing $X^{n}$ and $Y^{n}$ respectively, A and $\mathrm{B}$ send messages $M_{A}$ and $M_{B}$ using prefix free codes such that $E\left|M_{A}\right|=n R_{A}$ and $E\left|M_{B}\right|=n R_{B}$. Here $|$.$| denotes the length of the respective message$ in bits. $\mathrm{C}$ then broadcasts a message $M_{C}$ with $E\left|M_{C}\right|=n R_{C}$ to $\mathrm{A}$ and $\mathrm{B}$. Each of $\mathrm{A}$ and $\mathrm{B}$ then decode $f\left(X_{i}, Y_{i}\right) ; i=1,2, \cdots, n$ from the information available to them. For the relay network, a $\left(2^{n R_{A}}, 2^{n R_{B}}, 2^{n R_{C}}, n\right)$ variable length scheme consists of three encoders

$$
\phi_{A}: \mathcal{X}^{n} \longrightarrow\{0,1\}^{*}, \quad \phi_{B}: \mathcal{Y}^{n} \longrightarrow\{0,1\}^{*}, \quad \phi_{C}: \phi_{A}\left(\mathcal{X}^{n}\right) \times \phi_{B}\left(\mathcal{Y}^{n}\right) \longrightarrow\{0,1\}^{*}
$$

and two decoders

$$
\begin{aligned}
& \psi_{A}: \mathcal{X}^{n} \times \phi_{C}\left(\phi_{A}\left(\mathcal{X}^{n}\right) \times \phi_{B}\left(\mathcal{Y}^{n}\right)\right) \longrightarrow \mathcal{Z}^{n}, \\
& \psi_{B}: \mathcal{Y}^{n} \times \phi_{C}\left(\phi_{A}\left(\mathcal{X}^{n}\right) \times \phi_{B}\left(\mathcal{Y}^{n}\right)\right) \longrightarrow \mathcal{Z}^{n} .
\end{aligned}
$$

Here $\{0,1\}^{*}$ denotes the set of all finite length binary sequences. Let us define $\hat{Z}_{A}^{n}=\psi_{A}\left(X^{n}, \phi_{C}\left(\phi_{A}\left(X^{n}\right), \phi_{B}\left(Y^{n}\right)\right)\right)$ and $\hat{Z}_{B}^{n}=\psi_{B}\left(Y^{n}, \phi_{C}\left(\phi_{A}\left(X^{n}\right), \phi_{B}\left(Y^{n}\right)\right)\right)$ to be the decoder outputs. The probability of error for a $n$ length scheme is defined as

$$
P_{e}^{(n)} \triangleq \operatorname{Pr}\left\{\left(\hat{Z}_{A}^{n}, \hat{Z}_{B}^{n}\right) \neq\left(Z^{n}, Z^{n}\right)\right\}
$$


The rate triple $\left(R_{A}, R_{B}, R_{C}\right)$ of a code is defined as

$$
\begin{aligned}
& R_{A}=\frac{1}{n} \sum_{x^{n}} \operatorname{Pr}\left(x^{n}\right)\left|\phi_{A}\left(x^{n}\right)\right| \\
& R_{B}=\frac{1}{n} \sum_{y^{n}} \operatorname{Pr}\left(y^{n}\right)\left|\phi_{B}\left(y^{n}\right)\right| \\
& R_{C}=\frac{1}{n} \sum_{\left(x^{n}, y^{n}\right)} \operatorname{Pr}\left(x^{n}, y^{n}\right)\left|\phi_{C}\left(\phi_{A}\left(x^{n}\right), \phi_{B}\left(y^{n}\right)\right)\right| .
\end{aligned}
$$

A rate triple $\left(R_{A}, R_{B}, R_{C}\right)$ is said to be achievable with zero-error if for any $\epsilon>0$, there exists a scheme with $P_{e}^{(n)}=0$ for a large enough $n$ such that $\frac{1}{n} E\left|M_{A}\right| \leq R_{A}+\epsilon, \frac{1}{n} E\left|M_{B}\right| \leq R_{B}+\epsilon$ and $\frac{1}{n} E\left|M_{C}\right| \leq R_{C}+\epsilon$. The rate region $\mathcal{R}_{(0)}^{R N}(f, X, Y)$ is the closure of the convex hull of all achievable rate triples. The above setup is known as restricted input setup in the literature.

We now define the function computation in the relay network under a stricter setting, known as the unrestricted input setup. A $\left(2^{n R_{A}}, 2^{n R_{B}}, 2^{n R_{C}}, n\right)$ code for unrestricted input setup consists of three encoders and two decoders which are defined as before. Let $\left(\psi_{A}(\cdot)\right)_{i}$ and $\left(\psi_{B}(\cdot)\right)_{i}$ denote the $i$-th components of $\psi_{A}(\cdot)$ and $\psi_{B}(\cdot)$ respectively. A scheme is called a unrestricted input scheme if for each $x^{n} \in \mathcal{X}^{n}, y^{n} \in \mathcal{Y}^{n}$, and $i=1,2, \cdots, n$,

$$
\left(\psi_{A}\left(x^{n}, \phi_{C}\left(\phi_{A}\left(x^{n}\right), \phi_{B}\left(y^{n}\right)\right)\right)\right)_{i}=f\left(x_{i}, y_{i}\right)
$$

and

$$
\left(\psi_{B}\left(y^{n}, \phi_{C}\left(\phi_{A}\left(x^{n}\right), \phi_{B}\left(y^{n}\right)\right)\right)\right)_{i}=f\left(x_{i}, y_{i}\right)
$$

if $\left(x_{i}, y_{i}\right) \in S_{X Y}$. Note that this is a stricter condition than $P_{e}^{(n)}=0$. A pair of vectors $\left(x^{n}, y^{n}\right)$ for which a component $\left(x_{i}, y_{i}\right)$ is outside the support set $S_{X Y}$, does not contribute to $P_{e}^{(n)}$, and thus in the original zeroerror problem setup, the decoders are also not required to correctly compute the other components. However, the unrestricted setup requires the decoders to compute the function correctly on all the components where $\left(x_{i}, y_{i}\right) \in$ $S_{X Y}$. Achievable rates and the rate region $\mathcal{R}_{(u)}^{R N}(f, X, Y)$ under the unrestricted setup are defined similarly as before.

Broadcast Function Network: For the broadcast function network shown in Fig. 2, a variable length code for the function computation problem consists of one encoder

$$
\phi_{C}: \mathcal{X}^{n} \times \mathcal{Y}^{n} \longrightarrow\{0,1\}^{*}
$$


and two decoders

$$
\begin{aligned}
& \psi_{A}: \phi_{C}\left(\mathcal{X}^{n} \times \mathcal{Y}^{n}\right) \times \mathcal{X}^{n} \longrightarrow \mathcal{Z}^{n} \\
& \psi_{B}: \phi_{C}\left(\mathcal{X}^{n} \times \mathcal{Y}^{n}\right) \times \mathcal{Y}^{n} \longrightarrow \mathcal{Z}^{n}
\end{aligned}
$$

The rate of a code is defined as $\frac{1}{n} \sum_{\left(x^{n}, y^{n}\right)} \operatorname{Pr}\left(x^{n}, y^{n}\right)\left|\phi_{C}\left(x^{n}, y^{n}\right)\right|$, and the outputs of the decoders are given by $\hat{Z}_{A}^{n}=\psi_{A}\left(X^{n}, \phi_{C}\left(X^{n}, Y^{n}\right)\right)$ and $\hat{Z}_{B}^{n}=\psi_{B}\left(Y^{n}, \phi_{C}\left(X^{n}, Y^{n}\right)\right)$. A rate $R$ is said to be achievable with zero-error if for any $\epsilon>0$, there is a code of some length $n$ with rate $R+\epsilon$ and $P_{e}^{(n)} \triangleq \operatorname{Pr}\left\{\left(\hat{Z}_{A}^{n}, \hat{Z}_{B}^{n}\right) \neq\left(Z^{n}, Z^{n}\right)\right\}=0$. The optimal zero-error rate $R_{(0)}^{*(B F N)}(f, X, Y)$ is defined as the infimum of the set of all achievable rates. Note that $R_{(0)}^{*(B F N)}(f, X, Y)$ is the optimal rate under restricted input setup.

\section{B. 6 -error function computation}

Relay Network: A fixed length $\left(2^{n R_{A}}, 2^{n R_{B}}, 2^{n R_{C}}, n\right)$ code for function computation in the relay network consists of three encoder maps

$$
\begin{aligned}
& \phi_{A}: \mathcal{X}^{n} \longrightarrow\left\{1,2, \cdots, 2^{n R_{A}}\right\} \\
& \phi_{B}: \mathcal{Y}^{n} \longrightarrow\left\{1,2, \cdots, 2^{n R_{B}}\right\} \\
& \phi_{C}: \phi_{A}\left(\mathcal{X}^{n}\right) \times \phi_{B}\left(\mathcal{Y}^{n}\right) \longrightarrow\left\{1,2, \cdots, 2^{n R_{C}}\right\}
\end{aligned}
$$

and two decoder maps as defined in (2), 3). A rate triple $\left(R_{A}, R_{B}, R_{C}\right)$ is said to be achievable with $\epsilon$-error if there exists a sequence of $\left(2^{n R_{A}}, 2^{n R_{B}}, 2^{n R_{C}}, n\right)$ codes such that probability of error $P_{e}^{(n)} \rightarrow 0$ as $n \rightarrow \infty$. The achievable rate region $\mathcal{R}_{(\epsilon)}^{R N}(f, X, Y)$ is the closure of the convex hull of all achievable rate triples.

Broadcast Function Network: For the broadcast function network, a $\left(2^{n R}, n\right)$ code consists of one encoder map

$$
\phi_{C}: \mathcal{X}^{n} \times \mathcal{Y}^{n} \longrightarrow\left\{1,2, \cdots, 2^{n R}\right\}
$$

and the two decoder maps as defined in (5), 6). A rate $R$ is said to be achievable with $\epsilon$-error if there exists a sequence of $\left(2^{n R}, n\right)$ codes for which $P_{e}^{(n)} \rightarrow 0$ as $n \rightarrow \infty$. The optimal broadcast rate $R_{(\epsilon)}^{*(B F N)}(f, X, Y)$ in this case is the infimum of the set of all achievable rates.

\begin{tabular}{|l|l|l|}
\hline & \multicolumn{1}{|c|}{ Zero-error } & \multicolumn{1}{|c|}{$\epsilon$-error } \\
\hline BFN-CSI - optimal rates & $\bullet R_{(B)}^{*(B F N)}(f, X, Y)$ & $\bullet R_{(\epsilon)}^{*(B F N)}(f, X, Y)$ \\
\hline RN - rate regions & $\bullet \mathcal{R}_{(0)}^{R N}(f, X, Y)$ & $\bullet \mathcal{R}_{(\epsilon)}^{R N}(f, X, Y)$ \\
& $\bullet \mathcal{R}_{(u)}^{R N}(f, X, Y)$ - For unrestricted i/p setup & \\
\hline
\end{tabular}

TABLE II: Notations for different rate regions 


\section{Graph theoretic definitions}

Let $G$ be a graph with vertex set $V(G)$ and edge set $E(G)$. For two graphs $G_{1}$ and $G_{2}$ with $V\left(G_{1}\right) \cap V\left(G_{2}\right)=\emptyset$, union graph $G_{1} \cup G_{2}$ is defined as the graph with vertex set $V\left(G_{1}\right) \cup V\left(G_{2}\right)$ and edge set $E\left(G_{1}\right) \cup E\left(G_{2}\right)$. If $V\left(G_{1}\right)=V\left(G_{2}\right)$, then the union graph is defined to be the graph with vertex set $V\left(G_{1}\right)$ and edge set $E\left(G_{1}\right) \cup E\left(G_{2}\right)$. A set $I \subseteq V(G)$ is called an independent set if no two vertices in $I$ are adjacent in $G$. Let $\Gamma(G)$ denote the set of all independent sets of $G$. A clique of a graph $G$ is a complete subgraph of $G$. A clique of the largest size is called a maximum clique. The number of vertices in a maximum clique is called clique number of $G$ and is denoted by $\omega(G)$. The chromatic number of $G$, denoted by $\chi(G)$, is the minimum number of colors required to color the graph $G$. A graph $G$ is said to be perfect if for any vertex induced subgraph $G^{\prime}$ of $G, \omega\left(G^{\prime}\right)=\chi\left(G^{\prime}\right)$. Note that the vertex disjoint union of perfect graphs is also perfect.

The $n$-fold OR product of $G$, denoted by $G^{\vee n}$, is defined by $V\left(G^{\vee n}\right)=(V(G))^{n}$ and $E\left(G^{\vee n}\right)=\left\{\left(v^{n}, v^{\prime n}\right)\right.$ : $\left(v_{i}, v_{i}^{\prime}\right) \in E(G)$ for some $\left.i\right\}$. The $n$-fold AND product of $G$, denoted by $G^{\wedge n}$, is defined by $V\left(G^{\wedge n}\right)=(V(G))^{n}$ and $E\left(G^{\wedge n}\right)=\left\{\left(v^{n}, v^{\prime n}\right): v^{n} \neq v^{\prime n}\right.$, and either $v_{i}=v_{i}^{\prime}$ or $\left(v_{i}, v_{i}^{\prime}\right) \in E(G)$ for all $\left.i\right\}$.

For a graph $G$ and a random variable $X$ taking values in $V(G),(G, X)$ represents a probabilistic graph. Chromatic entropy [10] of $(G, X)$ is defined as

$$
H_{\chi}(G, X)=\min \{H(c(X)): c \text { is a coloring of } G\} .
$$

Let $W$ be distributed over the power set of $\mathcal{X}$. The graph entropy [27], [28] of the probabilistic graph $(G, X)$ is defined as

$$
H_{G}(X)=\min _{X \in W \in \Gamma(G)} I(W ; X)
$$

where $\Gamma(G)$ is the set of all independent sets of $G$. Here the minimum is taken over all conditional distributions $p_{W \mid X}$ which are non-zero only for $X \in W$. The following result was shown in [10].

$$
\lim _{n \rightarrow \infty} \frac{1}{n} H_{\chi}\left(G^{\vee n}, X^{n}\right)=H_{G}(X)
$$

Let $T_{P_{X}, \epsilon}^{n}$ denote the $\epsilon$-typical set of length $n$ under the distribution $P_{X}$, and let $G^{\wedge n}\left(T_{P_{X}, \epsilon}^{n}\right)$ be the vertex induced subgraph of $G^{\wedge n}$ with vertex set $T_{P_{X}, \epsilon^{*}}^{n} \quad$ The complementary graph entropy of $(G, X)$ is defined as

$$
\bar{H}_{G}(X)=\lim _{\epsilon \rightarrow 0} \limsup _{n \rightarrow \infty} \frac{1}{n} \log _{2}\left\{\chi\left(G^{\wedge n}\left(T_{P_{X}, \epsilon}^{n}\right)\right)\right\} .
$$

Unlike graph entropy, no single-letter characterization of the complementary graph entropy is known. It was shown 
in [11] that

$$
\lim _{n \rightarrow \infty} \frac{1}{n} H_{\chi}\left(G^{\wedge n}, X^{n}\right)=\bar{H}_{G}(X)
$$

The definition of graph entropy was extended to the conditional graph entropy in [3]. For a pair of random variables $(X, Y)$ and for a graph $G$ defined on the support set of $X$, the conditional graph entropy of $X$ given $Y$ is defined as

$$
H_{G}(X \mid Y)=\min _{\substack{W-X-Y \\ X \in W \in \Gamma(G)}} I(W ; X \mid Y)
$$

where the minimization is over all conditional distribution $p_{W \mid X}\left(=p_{W \mid X, Y}\right)$ which is non-zero only for $X \in W$.

We now define some graphs suitable for addressing our problem. For a function $f(x, y)$ defined over $\mathcal{X} \times \mathcal{Y}$, we define a graph called $f$-modified rook's graph. A rook's graph $G_{\mathcal{X} \mathcal{Y}}$ over $\mathcal{X} \times \mathcal{Y}$ is defined by the vertex set $\mathcal{X} \times \mathcal{Y}$ and edge set $\left\{\left((x, y),\left(x^{\prime}, y^{\prime}\right)\right): x=x^{\prime}\right.$ or $y=y^{\prime}$, but $\left.(x, y) \neq\left(x^{\prime}, y^{\prime}\right)\right\}$.

Definition 2 For a function $f(x, y)$ the $f$-modified rook's graph $R G_{X Y}^{f}$ has its vertex set $\mathcal{X} \times \mathcal{Y}$, and two vertices $\left(x_{1}, y_{1}\right)$ and $\left(x_{2}, y_{2}\right)$ are adjacent if and only if $\left.i\right)$ they are adjacent in the rook's graph $\left.G \mathcal{X} \mathcal{Y}, i i\right)\left(x_{1}, y_{1}\right),\left(x_{2}, y_{2}\right) \in$ $S_{X Y}$, and iii) $f\left(x_{1}, y_{1}\right) \neq f\left(x_{2}, y_{2}\right)$.

$f$-confusability graph $G_{X \mid Y}^{f}$ of $X, Y$ and $f$ was used in [3], [15] to study some function computation problems. Its vertex set is $\mathcal{X}$, and two vertices $x$ and $x^{\prime}$ are adjacent if and only if $\exists y \in \mathcal{Y}$ such that $f(x, y) \neq f\left(x^{\prime}, y\right)$ and $(x, y),\left(x^{\prime}, y\right) \in S_{X Y} \cdot G_{Y \mid X}^{f}$ is defined similarly.

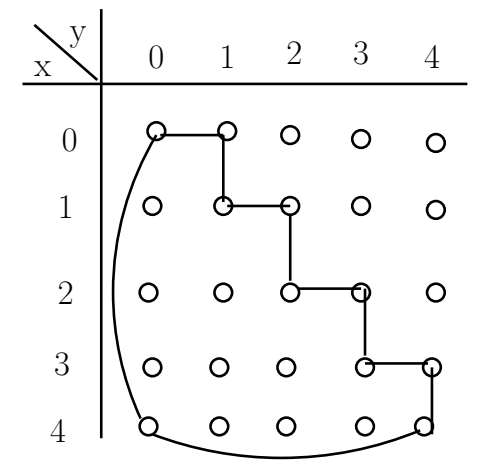

(a) $f$-modified rook's graph for $f(x, y)$ in (11)

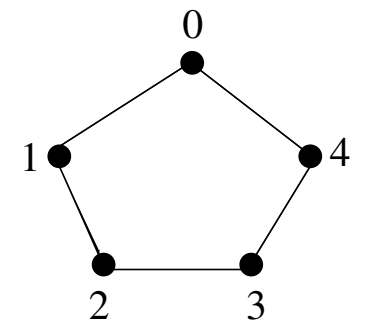

(b) $f$-confusability graphs $G_{X \mid Y}^{f}, G_{Y \mid X}^{f}$ for $f(x, y)$ in 11

Fig. 3: $f$-modified rook's graph and $f$-confusability graph

Example 1 Let us consider $X, Y \in\{0,1,2,3,4\}$ with distribution

$$
p(x, y)=\left\{\begin{array}{cl}
\frac{1}{10} & \text { if } y=x \text { or } y=x+1 \bmod 5 \\
0 & \text { otherwise }
\end{array}\right.
$$


and the equality function

$$
f(x, y)= \begin{cases}1 & \text { if } x=y \\ 0 & \text { if } x \neq y\end{cases}
$$

The f-modified rook's graph for this function is shown in Fig. $3 a$ Both $G_{X \mid Y}^{f}$ and $G_{Y \mid X}^{f}$ are the pentagon graph which is shown in Fig. $3 b$

Next we extend the definition of $R G_{X Y}^{f}$ to $n$ instances:

Definition $3 R G_{X Y}^{f}(n)$ has its vertex set $\mathcal{X}^{n} \times \mathcal{Y}^{n}$, and two vertices $\left(x^{n}, y^{n}\right)$ and $\left(x^{\prime n}, y^{\prime n}\right)$ are adjacent if and only if

(i) $x^{n}=x^{\prime n}$ or $y^{n}=y^{\prime n}$,

(ii) $\operatorname{Pr}\left(x^{n}, y^{n}\right) \cdot \operatorname{Pr}\left(x^{\prime n}, y^{\prime n}\right)>0$,

(iii) $\exists$ an $i \in\{1, \cdots, n\}$ such that $f\left(x_{i}, y_{i}\right) \neq f\left(x_{i}^{\prime}, y_{i}^{\prime}\right)$.

To address the unrestricted input setup, we define the following graph for $n$ instances.

Definition $4 R G_{X Y}^{f,(u)}(n)$ has its vertex set $\mathcal{X}^{n} \times \mathcal{Y}^{n}$, and two vertices $\left(x^{n}, y^{n}\right)$ and $\left(x^{\prime n}, y^{\prime n}\right)$ are adjacent if and only if

(i) $x^{n}=x^{\prime n}$ or $y^{n}=y^{\prime n}$,

(ii) $\exists$ an $i \in\{1, \cdots, n\}$ such that $f\left(x_{i}, y_{i}\right) \neq f\left(x_{i}^{\prime}, y_{i}^{\prime}\right)$ and $\left(x_{i}, y_{i}\right),\left(x_{i}^{\prime}, y_{i}^{\prime}\right) \in S_{X Y}$.

It is easy to see that the graph $R G_{X Y}^{f}(n)$ is a subgraph of $R G_{X Y}^{f,(u)}(n)$. Note that for $n=1$, these two graphs are the same.

Consider a graph $G$ with vertex set $\mathcal{V}$, where $\mathcal{V}$ has a Cartesian representation given by a one-to-one mapping $\pi: \mathcal{V} \rightarrow \mathcal{X} \times \mathcal{Y}$. For such a graph, the chromatic entropy region was defined in [15] as follows. If $c_{1}$ and $c_{2}$ are two maps of $\mathcal{X}$ and $\mathcal{Y}$ into $\{0,1\}^{*}$ respectively, then $c_{1} \times c_{2}$ denotes the map given by $\left(c_{1} \times c_{2}\right)(x, y)=\left(c_{1}(x), c_{2}(y)\right)$. A triple $\left(c_{1}, c_{2}, c\right)$ of functions of respectively $\mathcal{X}, \mathcal{Y}, \mathcal{V}$ into $\{0,1\}^{*}$ is called a color cover for $G$ if

i) $\left(c_{1} \times c_{2}\right) \circ \pi$ and $c$ are colorings of $G$.

ii) $c_{1} \times c_{2}$ is a refinement of $c$, i.e., $\exists$ a mapping $\theta:\left(c_{1} \times c_{2}\right)(\mathcal{X} \times \mathcal{Y}) \rightarrow\{0,1\}^{*}$ such that $\theta \circ\left(c_{1} \times c_{2}\right)=c$.

Let $\mathcal{C}$ denote the set of all color covers for $G$. For a probabilistic graph $(G, V)$, with vertex set $\mathcal{V}$ having a Cartesian representation $\pi: \mathcal{V} \rightarrow \mathcal{X} \times \mathcal{Y}$, let us denote $(X, Y)=\pi(V)$. Chromatic entropy region is defined as

$$
H_{\chi}(G, V, \pi) \triangleq \bigcup_{\left(c_{1}, c_{2}, c\right) \in \mathcal{C}}\left\{\left(b_{1}, b_{2}, b\right): b_{1} \geq H\left(c_{1}(X)\right), b_{2} \geq H\left(c_{2}(Y)\right), b \geq H(c(V))\right\}
$$


Graph entropy region was defined in [15] from the definition of chromatic entropy region as follows,

$$
H(G, V, \pi) \triangleq \bigcup_{n} \frac{1}{n} H_{\chi}\left(G^{n}, V^{n}, \pi^{n}\right),
$$

where $G^{n}$ denotes the $n$-fold OR product graph of $G$.

Let $R_{\chi}\left(R G_{X Y}^{f}, X, Y\right)$ denote the chromatic entropy region for $f$-modified rook's graph $R G_{X Y}^{f}$. Motivated from the graph entropy region, we define the following three dimensional regions for $f$-modified rook's graph

$$
\begin{aligned}
& Z_{X, Y}^{f} \triangleq \bigcup_{n} \frac{1}{n} R_{\chi}\left(R G_{X Y}^{f}(n), X^{n}, Y^{n}\right), \\
& Z_{X, Y}^{f,(u)} \triangleq \bigcup_{n} \frac{1}{n} R_{\chi}\left(R G_{X Y}^{f,(u)}(n), X^{n}, Y^{n}\right) .
\end{aligned}
$$

The graph in the following definition is used to give an inner bound for the zero-error computation in the relay network (Theorem 3).

Definition 5 Let $U_{1}$ and $U_{2}$ be two random variables such that $X \in U_{1} \in \Gamma\left(G_{X \mid Y}^{f}\right)$ and $Y \in U_{2} \in \Gamma\left(G_{Y \mid X}^{f}\right)$. The random variable $\left(U_{1}, U_{2}\right)$ over $\mathcal{U}_{1} \times \mathcal{U}_{2}$ has joint distribution with $(X, Y)$ as $p_{X, U_{1}, Y, U_{2}}\left(x, u_{1}, y, u_{2}\right)=$ $p(x, y) p\left(u_{1} \mid x\right) p\left(u_{2} \mid y\right)$. We define a graph $\widetilde{R G}_{U_{1} U_{2}}^{f}$ with vertex set $\mathcal{U}_{1} \times \mathcal{U}_{2}$. Two vertices $\left(u_{1}, u_{2}\right)$ and $\left(u_{1}^{\prime}, u_{2}^{\prime}\right)$ in $\widetilde{R G}_{U_{1} U_{2}}^{f}$ are connected if $\exists(x, y)$ and $\left(x^{\prime}, y^{\prime}\right)$ such that

1) $p_{X U_{1} Y U_{2}}\left(x, u_{1}, y, u_{2}\right), p_{X U_{1} Y U_{2}}\left(x^{\prime}, u_{1}^{\prime}, y^{\prime}, u_{2}^{\prime}\right)>0$,

2) $x=x^{\prime}, u_{1}=u_{1}^{\prime}$ and $f(x, y) \neq f\left(x^{\prime}, y^{\prime}\right)$

or

$y=y^{\prime}, u_{2}=u_{2}^{\prime}$ and $f(x, y) \neq f\left(x^{\prime}, y^{\prime}\right)$.

Note that by Definition 5 , two nodes $\left(u_{1}, u_{2}\right)$ and $\left(u_{1}^{\prime}, u_{2}^{\prime}\right)$ are connected in $\widetilde{R G_{U_{1} U_{2}}} f$ only if either $u_{1}=u_{1}^{\prime}$ or $u_{2}=u_{2}^{\prime}$, i.e., all connections are either row wise or column wise. Next we give an example to illustrate the above definitions. The function in Example 2 was used in [3] to explain the conditional graph entropy. Let us consider the same function for our function computation problem in the relay network.

Example 2 [3] Consider $X, Y \in\{1,2,3\}$

$$
p(x, y)= \begin{cases}\frac{1}{6} & \text { if } x \neq y \\ 0 & \text { otherwise }\end{cases}
$$

and

$$
f(x, y)= \begin{cases}1 & \text { if } x>y \\ 0 & \text { if } x \leq y\end{cases}
$$


Both the confusability graphs are the same graph which is shown in Fig. 4a The f-modified rook's graph for this function is shown in Fig. $4 b$

In Example 2, the distribution of $(X, Y)$ is symmetric in $X$ and $Y$ and the function values are also symmetric. For this example, let us consider an instance of $U_{1}$ and $U_{2}$ as follows. Let $\mathcal{U}_{1}$ be $\{\{1,2\},\{2,3\}\}$ and let us denote it by $\{a, b\}$ where $a=\{1,2\}$ and $b=\{2,3\}$. Similarly, we choose $\mathcal{U}_{2}$ and we denote it by $\{c, d\}$, where $c=\{1,2\}$ and $d=\{2,3\}$. The conditional distributions are given by $p_{U_{1} \mid X}(a \mid 2)=p_{U_{1} \mid X}(b \mid 2)=p_{U_{2} \mid Y}(c \mid 2)=p_{U_{2} \mid Y}(d \mid 2)=\frac{1}{2}$. Now let us consider the graph $\widetilde{R G}_{U_{1} U_{2}}^{f}$ for this function. The nodes $(a, c)$ and $(a, d)$ are connected in $\widetilde{R G}_{U_{1} U_{2}}^{f}$ because $p_{X U_{1} Y U_{2}}(2, a, 1, c), p_{X U_{1} Y U_{2}}(2, a, 3, d)>0$ and $f(2,1) \neq f(2,3)$. By considering other pairs of nodes in $\widetilde{R G}_{U_{1} U_{2}}^{f}$, we can verify that the graph $\widetilde{R G}_{U_{1} U_{2}}^{f}$ is a "square" graph which is shown in Fig. $4 \mathrm{c}$.

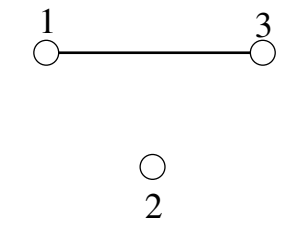

(a) Graphs $G_{X \mid Y}^{f}, G_{Y \mid X}^{f}$

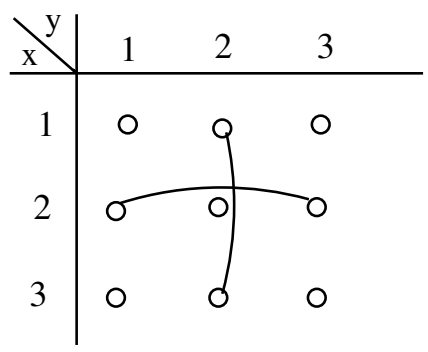

(b) Graph $R G_{X Y}^{f}$

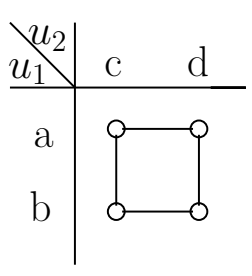

(c) Graph $\widetilde{R G}_{U_{1} U_{2}}^{f}$

Fig. 4: Graphs for Example 2

\section{RESULTS}

\section{A. Results for zero-error computation}

We first give the results for the broadcast function computation problem shown in Fig. 2, For this problem, we show that the optimal rate under zero-error and $\epsilon$-error are the same. Proofs of all the theorems in this subsection are given in Section IV

Theorem 1 For the broadcast function computation problem with complementary side information shown in Fig. 2 the optimal zero-error broadcast rate $R_{(0)}^{*(B F N)}(f, X, Y)$ for computing $Z=f(X, Y)$ is given by

$$
R_{(0)}^{*(B F N)}(f, X, Y)=\max \{H(Z \mid X), H(Z \mid Y)\}
$$

Computing a CWOOF in the relay network is equivalent to exchanging $X$ and $Y$. Hence using Theorem 11, we get a single-letter characterization for computing component-wise one-to-one function in the relay network (Fig. 1) when the support set $S_{X Y}$ is the full set. 
Corollary 1 (CWOOF in $\mathbf{R N})$ If $S_{X Y}=\mathcal{X} \times \mathcal{Y}$, then the zero-error rate region for computing a component-wise one-to-one function at nodes $A$ and $B$ in the relay network is given by

$$
\mathcal{R}_{(0)}^{R N}(f, X, Y) \triangleq\left\{\left(R_{A}, R_{B}, R_{C}\right): R_{A} \geq H(X), R_{B} \geq H(Y), R_{C} \geq \max \{H(Y \mid X), H(X \mid Y)\}\right\}
$$

We note that the problem of exchanging $X$ and $Y$ through a relay has been addressed in [7] under $\epsilon$-error criteria. The rate region for this problem under the $\epsilon$-error criteria is given by

$$
\left\{\left(R_{A}, R_{B}, R_{C}\right): R_{A} \geq H(X \mid Y), R_{B} \geq H(Y \mid X), R_{C} \geq \max \{H(Y \mid X), H(X \mid Y)\}\right\}
$$

When the sources are independent, the rate regions are clearly the same under $\epsilon$-error and zero-error criteria. When the sources are dependent with full support, smaller rates are possible for $R_{A}$ and $R_{B}$ under $\epsilon$-error compared to zero-error. Even in this case, the minimum possible rate for $R_{C}$ is the same in both the cases.

Theorem 2 (RN, multiletter characterization) (a) The zero-error rate region is given by, $\mathcal{R}_{(0)}^{R N}(f, X, Y)=Z_{X, Y}^{f}$.

(b) The rate region under unrestricted input setup is given by, $\mathcal{R}_{(u)}^{R N}(f, X, Y)=Z_{X, Y}^{f,(u)}$,

where $Z_{X, Y}^{f}$ and $Z_{X, Y}^{f,(u)}$ are as defined in (13) and (14) respectively.

Since a scheme under the unrestricted input setup is also a zero-error scheme, $\mathcal{R}_{(u)}^{R N}(f, X, Y) \subseteq \mathcal{R}_{(0)}^{R N}(f, X, Y)$. The multi letter expressions for the rate regions given in Theorem 2 are difficult to compute. We give a single-letter inner bound for $\mathcal{R}_{(u)}^{R N}(f, X, Y)$ in Theorem 3. This bound is proved by considering the problem under unrestricted input setup. Our proof technique is similar to the ones in [15].

\section{Theorem 3 (RN, zero-error inner bound)}

(a) Let

$$
\begin{aligned}
\mathcal{R}_{I} \triangleq\left\{\left(R_{A}, R_{B}, R_{C}\right):\right. & R_{A} \geq I\left(X ; U_{1} \mid Q\right), R_{B} \geq I\left(Y ; U_{2} \mid Q\right), \\
& \left.R_{C} \geq \min \left\{I\left(W ; U_{1}, U_{2} \mid Q\right), \max \left\{I\left(X ; U_{1} \mid Q\right), I\left(Y ; U_{2} \mid Q\right)\right\}\right\}\right\}
\end{aligned}
$$

for some $p(q) p\left(w \mid u_{1}, u_{2}, q\right) p\left(u_{1} \mid x, q\right) p\left(u_{2} \mid y, q\right)$ such that

(i) $X \in U_{1} \in \Gamma\left(G_{X \mid Y}^{f}\right)$

(ii) $Y \in U_{2} \in \Gamma\left(G_{Y \mid X}^{f}\right)$

(iii) $\left(U_{1}, U_{2}\right) \in W \in \Gamma\left(\widetilde{R G}_{U_{1} U_{2}}^{f}\right)$.

Then $\mathcal{R}_{I} \subseteq \mathcal{R}_{(u)}^{R N}(f, X, Y)$.

(b) The two upper bounds for $R_{C}$ above, namely $I\left(W ; U_{1}, U_{2}\right)$ and $\max \left\{I\left(X ; U_{1}\right), I\left(Y ; U_{2}\right)\right\}$, are not comparable in general. 
The proof of Theorem 3 is given in Section $\mathrm{IV}-\mathrm{C}$. To prove part (b), we show the following. For the function computation problem in Example 1. $\exists\left(U_{1}^{\prime}, U_{2}^{\prime}, W^{\prime}\right)$ s.t. $I\left(W^{\prime} ; U_{1}^{\prime}, U_{2}^{\prime}\right)<\max \left\{I\left(X ; U_{1}\right), I\left(Y ; U_{2}\right)\right\}$ for any $\left(U_{1}, U_{2}\right)$, and for the function computation problem in Example $2 \exists\left(U_{1}^{\prime}, U_{2}^{\prime}\right)$ s.t. $\max \left\{I\left(X ; U_{1}^{\prime}\right), I\left(Y ; U_{2}^{\prime}\right)\right\}<I\left(W ; U_{1}, U_{2}\right)$ for any $\left(U_{1}, U_{2}, W\right)$.

The following corollary follows from Theorem 3 .

Corollary 2 Any rate triple $\left(R_{A}, R_{B}, R_{C}\right)$ such that

$$
R_{A} \geq H_{G_{X \mid Y}^{f}}(X), R_{B} \geq H_{G_{Y \mid X}^{f}}(Y), R_{C} \geq \max \left\{H_{G_{X \mid Y}^{f}}(X), H_{G_{Y \mid X}^{f}}(Y)\right\}
$$

is achievable.

Next we provide a sufficient condition on the joint distribution $p_{X Y}$ under which the relay can also compute the function whenever nodes A and B compute it with zero-error.

Theorem 4 (RN, relay's knowledge) If $p(x, y)>0 \forall(x, y) \in \mathcal{X} \times \mathcal{Y}$, then for any zero-error scheme the relay can also compute the function with zero-error.

Theorem 4 does not hold if $S_{X Y} \neq \mathcal{X} \times \mathcal{Y}$. We show an instance of encoding for the function given in Example 2 to demonstrate this. Let $\phi_{A}, \phi_{B}$ and $\phi_{C}$ be as follows.

$$
\begin{gathered}
\phi_{A}= \begin{cases}1 & \text { if } x=1 \\
0 & \text { otherwise. }\end{cases} \\
\phi_{B}= \begin{cases}1 & \text { if } y=1 \\
0 & \text { otherwise. }\end{cases} \\
\phi_{C}= \begin{cases}1 & \text { if } \phi_{A}=\phi_{B} \\
0 & \text { otherwise. }\end{cases}
\end{gathered}
$$

Here nodes $\mathrm{A}$ and $\mathrm{B}$ recover the function with zero-error, but the relay can not reconstruct the function. When $\phi_{A}=\phi_{B}=0((x, y)$ is either $(2,3)$ or $(3,2))$, the function value can be both 0 and 1 . So $H\left(f \mid \phi_{A}, \phi_{B}\right)>0$.

\section{B. Results for $\epsilon$-error computation}

In this section, we give our results for $\epsilon$-error function computation in the relay network (RN). Using Lemma 9 given in the appendix, we can observe that in $\mathrm{RN}$, if a rate triple $\left(R_{A}, R_{B}, R_{C}\right)$ is achievable under zero-error, then $\left(R_{A}+\delta, R_{B}+\delta, R_{C}+\delta\right)$ is achievable under $\epsilon$-error for any $\delta>0$. This shows that in general the rate region for computing a function in $\mathrm{RN}$ with $\epsilon$-error is equal to or larger than the rate region for computing the function 
with zero-error. In Example 3, we give an instance for which the rate region under $\epsilon$-error is strictly larger than the rate region under zero-error. Proofs of all the theorems in this subsection are given in Section $\mathrm{V}$.

Example 3 Let us consider computing $X \oplus Y$ for a doubly symmetric binary source $(D S B S(p))(X, Y)$ where $p_{X, Y}(0,0)=p_{X, Y}(1,1)=(1-p) / 2$ and $p_{X, Y}(0,1)=p_{X, Y}(1,0)=p / 2$. From Corollary 1 we have the zeroerror rate region as $\left\{\left(R_{A}, R_{B}, R_{C}\right): R_{A} \geq 1, R_{B} \geq 1, R_{C} \geq H(p)\right\}$. As noted before, computing $X \oplus Y$ in the relay network is same as exchanging $X$ and $Y$. The $\epsilon$-error rate region for exchanging $X$ and $Y$ through the relay is given in (15). Computing this for $\operatorname{DSBS}(p)(X, Y)$ gives the rate region as $\left\{\left(R_{A}, R_{B}, R_{C}\right): R_{A}, R_{B}, R_{C} \geq H(p)\right\}$.

For arbitrary functions, we do not have a single-letter characterization for the $\epsilon$-error rate region. Next lemma gives a cutset outer bound for the $\epsilon$-error rate region.

Lemma 1 (a) [Cutset outer bound] Any achievable rate triple $\left(R_{A}, R_{B}, R_{C}\right) \in \mathcal{R}_{(\epsilon)}^{R N}(f, X, Y)$ for $R N$ satisfies the following :

$$
R_{A} \geq H_{G_{X \mid Y}^{f}}(X \mid Y), \quad R_{B} \geq H_{G_{Y \mid X}^{f}}(Y \mid X), \quad R_{C} \geq \max \{H(Z \mid X), H(Z \mid Y)\}
$$

(b) Equality in (16) can be achieved individually for either $\left(R_{A}, R_{B}\right)$ or $R_{C}$.

Remark 1 We suspect the cutset bound to be loose, though we do not have an example to show this. For all the example functions where we have a single-letter characterization of the rate region, the cutset outer bound in (16) is seen to be tight. Example 4 provides a class of functions for which the cutset outer bound is tight.

Next we propose two achievable schemes for the $\epsilon$-error computation problem. These two schemes are the extensions of the zero-error schemes given in Theorem 3 .

Theorem 5 (RN, $\epsilon$-error inner bound) (a) Let

$$
\begin{aligned}
\mathcal{R}_{I 1}^{\epsilon} \triangleq & \left\{\left(R_{A}, R_{B}, R_{C}\right): R_{A} \geq I\left(X ; U_{1} \mid U_{2}, Q\right), R_{B} \geq I\left(Y ; U_{2} \mid U_{1}, Q\right),\right. \\
& \left.R_{A}+R_{B} \geq I\left(X, Y ; U_{1}, U_{2} \mid Q\right), R_{C} \geq \max \left\{I\left(W ; U_{1} \mid U_{2}, Y, Q\right), I\left(W ; U_{2} \mid U_{1}, X, Q\right)\right\}\right\}
\end{aligned}
$$

for some $p(q) p\left(w \mid u_{1}, u_{2}, q\right) p\left(u_{1} \mid x, q\right) p\left(u_{2} \mid y, q\right)$ such that

(i) $X \in U_{1} \in \Gamma\left(G_{X \mid Y}^{f}\right)$

(ii) $Y \in U_{2} \in \Gamma\left(G_{Y \mid X}^{f}\right)$

(iii) $\left(U_{1}, U_{2}\right) \in W \in \Gamma\left(\widetilde{R G}_{U_{1} U_{2}}^{f}\right)$. 
Let

$$
\begin{aligned}
\mathcal{R}_{I 2}^{\epsilon} \triangleq\left\{\left(R_{A}, R_{B}, R_{C}\right):\right. & R_{A} \geq H_{G_{X \mid Y}^{f}}(X \mid Y), R_{B} \geq H_{G_{Y \mid X}^{f}}(Y \mid X), \\
& \left.R_{C} \geq \max \left\{H_{G_{X \mid Y}^{f}}(X \mid Y), H_{G_{Y \mid X}^{f}}(Y \mid X)\right\}\right\} .
\end{aligned}
$$

Let $\mathcal{R}_{I}^{\epsilon}$ be the convex closure of $\mathcal{R}_{I 1}^{\epsilon} \cup \mathcal{R}_{I 2}^{\epsilon}$. Then $\mathcal{R}_{I}^{\epsilon} \subseteq \mathcal{R}_{(\epsilon)}^{R N}(f, X, Y)$.

(b) Neither of $\mathcal{R}_{I 1}^{\epsilon}$ and $\mathcal{R}_{I 2}^{\epsilon}$ is a subset of the other in general.

The proof of Theorem 5 is given in Section $\mathrm{V}-\mathrm{A}$. To prove part (b), we show that for computing AND for a $D S B S(p)$ source, the rate triple $(H(p), H(p), H(p)) \in \mathcal{R}_{I 2}^{\epsilon} \backslash \mathcal{R}_{I 1}^{\epsilon}$, and $\left(1, H(p), \frac{1}{2} H(p)\right) \in \mathcal{R}_{I 1}^{\epsilon} \backslash \mathcal{R}_{I 2}^{\epsilon}$.

Example 4 Let us consider the functions where one of the confusability graphs is empty. W.l.o.g., let us assume that $G_{Y \mid X}^{f}$ is empty. Then on the support set $S_{X Y}$, the function $f$ can be computed from $X$ alone. This implies that node $A$ can compute the function with zero-error from $X$, and $H_{G_{Y \mid X}^{f}}(Y \mid X)=0$. Let us consider $H_{G_{X \mid Y}^{f}}(X \mid Y)$. In general, $H_{G_{X \mid Y}^{f}}(X \mid Y) \geq H(Z \mid Y)$. For a given $Z=z$, let us consider the set of all $x, A_{z}=\{x: f(x, y)=$ $z$, for some $y$ s.t. $\left.(x, y) \in S_{X Y}\right\}$. Since here for $X=x, f\left(x, y^{\prime}\right)=f\left(x, y^{\prime \prime}\right)$ for any $\left(x, y^{\prime}\right),\left(x, y^{\prime \prime}\right) \in S_{X Y}, A_{z}$ is an independent set of $G_{X \mid Y}^{f}$. Let $\mathcal{A}$ denote the set of all $A_{z}$, and $W=A_{Z}$. Since $Z$ is a function of $X$, we have $W=g(X)$ for some function $g$. This $W$ in 10 gives that $I(W ; X \mid Y)=H(Z \mid Y)$. So we get $H_{G_{X \mid Y}^{f}}(X \mid Y)=$ $H(Z \mid Y)$. Then we get $\mathcal{R}_{I 2}^{\epsilon}$ in Theorem 5 as $\left\{\left(R_{A}, R_{B}, R_{C}\right): R_{A} \geq H(Z \mid Y), R_{B} \geq 0, R_{C} \geq H(Z \mid Y)\right\}$. It is easy to check that the cutset outer bound in 16 also gives the same rate region. This shows that for functions where one of the confusability graph is empty, the cutset outer bound is tight.

Theorem 6 Let $f_{1}, f_{2}$ be two functions of $(X, Y)$.

(a) If $E\left(R G_{X Y}^{f_{1}}\right) \subseteq E\left(R G_{X Y}^{f_{2}}\right)$, then $(i) \mathcal{R}_{(0)}^{R N}\left(f_{1}, X, Y\right) \supseteq \mathcal{R}_{(0)}^{R N}\left(f_{2}, X, Y\right),(i i) \mathcal{R}_{(\epsilon)}^{R N}\left(f_{1}, X, Y\right) \supseteq \mathcal{R}_{(\epsilon)}^{R N}\left(f_{2}, X, Y\right)$.

(b) If $R G_{X Y}^{f_{1}}$ is isomorphic to $R G_{X Y}^{f_{2}}$, then $(i) \mathcal{R}_{(0)}^{R N}\left(f_{1}, X, Y\right)=\mathcal{R}_{(0)}^{R N}\left(f_{2}, X, Y\right),(i i) \mathcal{R}_{(\epsilon)}^{R N}\left(f_{1}, X, Y\right)=\mathcal{R}_{(\epsilon)}^{R N}\left(f_{2}, X, Y\right)$.

For any arbitrary function $f$ of $(X, Y)$, if $R G_{X Y}^{f}$ is isomorphic to the the $f$-modified rook's graph for exchanging $X$ and $Y$ (i.e. computing a component-wise one-to-one function ), then the rate region $\mathcal{R}_{(\epsilon)}^{R N}(f, X, Y)$ is given by (15). $\mathcal{R}_{(\epsilon)}^{R N}\left(f_{1}, X, Y\right)=\mathcal{R}_{(\epsilon)}^{R N}\left(f_{2}, X, Y\right)$ does not imply the isomorphism between $R G_{X Y}^{f_{1}}$ and $R G_{X Y}^{f_{2}}$. We show this through the following example.

Example 5 For a $D S B S(p)(X, Y)$, let functions $f_{1}, f_{2}$ of $(X, Y)$ be defined as ${ }^{*} f_{1}=X+Y$ and $f_{2}=Y \cdot(X+Y)$. For these functions, $R G_{X Y}^{f_{1}}$ and $R G_{X Y}^{f_{2}}$ are shown in Fig. 5 The graph $R G_{X Y}^{f_{1}}$ is same as as the $f$-modified rook's graph for computing a component-wise one-to-one function. Using Theorem 6 we get $\mathcal{R}_{(\epsilon)}^{R N}\left(f_{1}, X, Y\right)=$ $\left\{\left(R_{A}, R_{B}, R_{C}\right): R_{A}, R_{B}, R_{C} \geq H(p)\right\}$. For function $f_{2}$, since graphs $G_{X \mid Y}^{f_{2}}$ and $G_{Y \mid X}^{f_{2}}$ are complete graphs, $H_{G_{X \mid Y}^{f_{2}}}(X \mid Y)=H(X \mid Y)$, and $H_{G_{Y \mid X}^{f_{2}}}(Y \mid X)=H(Y \mid X)$. Further, we have $H\left(Z_{2} \mid X\right)=H(p)$ and $H\left(Z_{2} \mid Y\right)=$ ${ }^{*}$ Here + is sum, not XOR. In particular, $f_{1}(1,1)=2$. 
$\frac{1}{2} H(p)$. This implies that $\max \left\{H_{G_{X \mid Y}^{f}}(X \mid Y), H_{G_{Y \mid X}^{f}}(Y \mid X)\right\}=\max \{H(Z \mid X), H(Z \mid Y)\}=H(p)$. Then the region given by $\mathcal{R}_{I 2}^{\epsilon}$ in Theorem 5 is same as the region given by the cutset outer bound in $\left[16\right.$. So we get $\mathcal{R}_{(\epsilon)}^{R N}\left(f_{2}, X, Y\right)=$ $\left\{\left(R_{A}, R_{B}, R_{C}\right): R_{A}, R_{B}, R_{C} \geq H(p)\right\}$ which is same as $\mathcal{R}_{(\epsilon)}^{R N}\left(f_{1}, X, Y\right)$. Here, even though $\mathcal{R}_{(\epsilon)}^{R N}\left(f_{1}, X, Y\right)=$ $\mathcal{R}_{(\epsilon)}^{R N}\left(f_{2}, X, Y\right), R G_{X Y}^{f_{1}}$ is not isomorphic to $R G_{X Y}^{f_{2}}$.

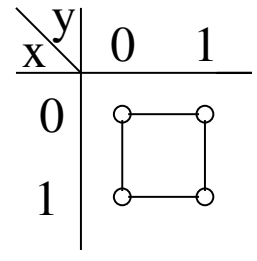

(a) $R G_{X Y}^{f_{1}}$

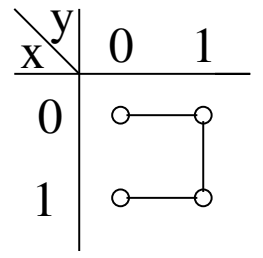

(b) $R G_{X Y}^{f_{2}}$

Fig. 5: Graphs $R G_{X Y}^{f_{1}}$ and $R G_{X Y}^{f_{2}}$ in Example 5

In [2], Han and Kobayashi considered the function computation problem where two encoders encode $X^{n}$ and $Y^{n}$, and a decoder wants to compute $f(X, Y)$ from the encoded messages. They gave necessary and sufficient conditions under which the function computation rate region coincides with the Slepian-Wolf region. The conditions were based on a probability-free structure of the function $f(X, Y)$, assuming that $S_{X Y}=\mathcal{X} \times \mathcal{Y}$. For our function computation problem, in general, if $R G_{X Y}^{f}$ is not the same as the $f$-modified rook's graph for a component-wise one-to-one function, then the equality $\mathcal{R}_{(\epsilon)}^{R N}(f, X, Y)=\mathcal{R}_{(\epsilon)}^{R N}(C W O O F, X, Y)$ also depends on $p_{X Y}$ even when $S_{X Y}=\mathcal{X} \times \mathcal{Y}$. In particular, for the function $f_{2}$ in Example 5 , the equality $\mathcal{R}_{(\epsilon)}^{R N}(f, X, Y)=\mathcal{R}_{(\epsilon)}^{R N}(C W O O F, X, Y)$ depends on the distribution $p_{X Y}$. This is illustrated in Example 6. Thus we observe that the characterization of $\mathcal{R}_{(\epsilon)}^{R N}(f, X, Y)=\mathcal{R}_{(\epsilon)}^{R N}(C W O O F, X, Y)$ in the relay network cannot have a probability-free structure.

Example 6 Let us consider the function $f_{2}$ in Example 5. When $p_{X Y}$ is DSBS( $\left.p\right)$, it is shown in Example 5 that $\mathcal{R}_{(\epsilon)}^{R N}(f, X, Y)=\mathcal{R}_{(\epsilon)}^{R N}(C W O O F, X, Y)$. Let us consider the same function for the following distribution

$$
\begin{aligned}
& p(0,0)=p(1,0)=\frac{1}{6}, \\
& p(0,1)=p(1,1)=\frac{1}{3} .
\end{aligned}
$$

We have $H(X \mid Y)=H(X)=1$ and $H(Y \mid X)=H(Y)=H\left(\frac{1}{3}\right)$. So we get $\mathcal{R}_{(\epsilon)}^{R N}(C W O O F, X, Y)=\left\{\left(R_{A}, R_{B}, R_{C}\right)\right.$ : $\left.R_{A} \geq 1, R_{B} \geq H\left(\frac{1}{3}\right), R_{C} \geq 1\right\}$. For $Z=f_{2}(X, Y), H(Z \mid Y)=\frac{2}{3}$, and $H(Z \mid X)=H(1 / 3) \approx 0.91$. Let us consider an instance of encoding where $A$ and $B$ communicate $X^{n}$ and $Y^{n}$ to the relay with rates $R_{A}=H(X)$ and $R_{B}=H(Y)$ respectively; and the relay computes $Z^{n}$ and use Slepian-Wolf binning to compress it at a rate $R_{C}=\max \{H(Z \mid X), H(Z \mid Y)\}$. Then the function computation at $A$ and $B$ follows from the Slepian-Wolf decoding. For this scheme, the rate triple $(1, H(1 / 3), H(1 / 3))$ is achievable. Clearly, $(1, H(1 / 3), H(1 / 3)) \notin$ $\mathcal{R}_{(\epsilon)}^{R N}(C W O O F, X, Y)$ and we get $\mathcal{R}_{(\epsilon)}^{R N}(f, X, Y) \neq \mathcal{R}_{(\epsilon)}^{R N}(C W O O F, X, Y)$. 


\section{ZERO ERROR COMPUTATION: PROOFS OF THEOREMS 1-4}

\section{A. Proof of Theorem 1}

The optimal $\epsilon$-error rate $R_{(\epsilon)}^{*(B F N)}(f, X, Y)$ is given by $\max \{H(Z \mid X), H(Z \mid Y)\}$ which follows from the SlepianWolf result [13]. Using Lemma 9, we can observe that $R_{(\epsilon)}^{*(B F N)}(f, X, Y) \leq R_{(0)}^{*(B F N)}(f, X, Y)$. Next we show that $R_{(0)}^{*(B F N)}(f, X, Y) \leq \max \{H(Z \mid X), H(Z \mid Y)\}$. The code has two constituents: a subset $S \subseteq \mathcal{X}^{n} \times \mathcal{Y}^{n}$, and a random binning of all sequences $z^{n}$ into $2^{R^{\prime} n}$ bins, where $R^{\prime}=\max \{H(Z \mid Y), H(Z \mid X)\}+\frac{\delta}{2}$. Let $S$ be the set of all $\left(x^{n}, y^{n}\right)$ satisfying at least one of the following:

E1: $\left(x^{n}, f\left(x^{n}, y^{n}\right)\right) \notin T_{\epsilon}^{n}(X Z)$,

E2: $\left(y^{n}, f\left(x^{n}, y^{n}\right)\right) \notin T_{\epsilon}^{n}(Y Z)$,

E3: $\exists z^{\prime n} \neq f\left(x^{n}, y^{n}\right)$ such that $\left(z^{\prime n}, x^{n}\right) \in T_{\epsilon}^{n}(Z X)$, and it is in the same bin as $f\left(x^{n}, y^{n}\right)$,

E4: $\exists z^{\prime n} \neq f\left(x^{n}, y^{n}\right)$ such that $\left(z^{\prime n}, y^{n}\right) \in T_{\epsilon}^{n}(Z Y)$, and it is in the same bin as $f\left(x^{n}, y^{n}\right)$.

The sequences in $S$ are indexed by a fixed length code of length at most $n(\log |\mathcal{X}|+\log |\mathcal{Y}|)$. The overall code consists of the indices of $S$ and the indices of the bins, distinguished by an additional prefix bit.

The encoder sends the bin index of $f\left(X^{n}, Y^{n}\right)$ if $\left(X^{n}, Y^{n}\right) \in S^{\complement}$. Otherwise, it sends the index of $\left(X^{n}, Y^{n}\right)$ in $S$. If node A receives a bin index, then it finds the unique $Z^{n}$ which is jointly typical with $X^{n}$. Otherwise, node A gets to know $\left(X^{n}, Y^{n}\right)$ from its index in $S$, and computes $Z^{n}=f\left(X^{n}, Y^{n}\right)$. Node B follows similar decoding. There is no decoding error either for node A or B under this scheme, as all sequences $\left(x^{n}, y^{n}\right)$ which could have resulted in a decoding error are separately transmitted using their index in $S$. From the Slepian-Wolf result [13], we know that the probability $\operatorname{Pr}(E 1 \cup E 3)$ is less than or equal to $2^{-n \delta / 2}$ for large enough $n$. Similarly, $\operatorname{Pr}(E 2 \cup E 4)$ is less than or equal to $2^{-n \delta / 2}$. Thus by union bound, $\operatorname{Pr}(S) \leq \operatorname{Pr}(E 1 \cup E 3)+\operatorname{Pr}(E 2 \cup E 4) \leq 2 \times 2^{-n \delta / 2}$. Since $\log |S|$ is linear in $n$, the overall average length of the code is at most

$$
\begin{aligned}
& \operatorname{Pr}(S) n(\log |\mathcal{X}|+\log |\mathcal{Y}|)+\operatorname{Pr}\left(S^{\complement}\right) n R^{\prime} \\
& \leq 2 \times 2^{-n \delta / 2} n(\log |\mathcal{X}|+\log |\mathcal{Y}|)+n\left(\max \{H(Z \mid Y), H(Z \mid X)\}+\frac{\delta}{2}\right) \\
& \leq n \frac{\delta}{2}+n\left(\max \{H(Z \mid Y), H(Z \mid X)\}+\frac{\delta}{2}\right) \\
& =n(\max \{H(Z \mid Y), H(Z \mid X)\}+\delta)
\end{aligned}
$$

for large enough $n$. This completes the proof of the theorem.

Proof of Corollary 17 First let us consider the converse for the rate region. For $R_{A}$, let us consider the cut between node A and a super node consisting of B and C. This situation arises when the relay node broadcasts the 
message sent by node A. Then the problem reduces to the problem of decoding with side information studied in [10], where the decoder with side information $Y$ wants to recover $X$. Lemma 6 in [10] shows that the optimal rate is equal to $\lim _{n \rightarrow \infty} \frac{1}{n} H_{\chi}\left(G^{\wedge n}, X^{n}\right)=\bar{H}_{G}(X)$. Since the support set is full, the graph $(G, X)$ is a complete graph with vertex set $\mathcal{X}$. It can be easily verified that for a complete graph, $\bar{H}_{G}(X)=H(X)$. So here we get $R_{A} \geq H(X)$.

Similarly, $R_{B} \geq H(Y)$. Now let us consider the rate $R_{C}$. Any relay encoding $\phi_{C}\left(\phi_{A}\left(x^{n}\right), \phi_{B}\left(y^{n}\right)\right)$ is also a function of $\left(x^{n}, y^{n}\right)$ and so any achieved rate $R_{C}$ can also be achieved if the relay has the full information $\left(x^{n}, y^{n}\right)$. So the optimum $R_{C}$ attains its minimum value when the relay has $X$ and $Y$. For a component-wise one-to-one function $Z, H(Z \mid X)=H(Y \mid X)$ and $H(Z \mid Y)=H(X \mid Y)$. Theorem 1 shows that if relay has both $X$ and $Y$, the minimum achievable broadcast rate is $\max \{H(Y \mid X), H(X \mid Y)\}$. This completes the converse. Now let us consider a scheme where nodes $\mathrm{A}$ and $\mathrm{B}$ communicate $X$ and $Y$ respectively to the relay. The relay can recover $X$ and $Y$ with zero-error if $R_{A}>H(X)$ and $R_{B}>H(Y)$. If the relay has $X$ and $Y$, Theorem 1 shows that the rate $\max \{H(Y \mid X), H(X \mid Y)\}$ is achievable for $R_{C}$ for computing a component-wise one-to-one function. This proves the achievability of the rate region.

\section{B. Proof of Theorem 2}

To prove Theorem 2, we first present some lemmas.

Lemma 2 For any $n \geq 1$, and given the encoding functions $\phi_{A}, \phi_{B}, \phi_{C}$, the nodes $A$ and $B$ can recover $f\left(X^{n}, Y^{n}\right)$ with zero-error if and only if $\phi_{C} \circ\left(\phi_{A} \times \phi_{B}\right)$ is a coloring of $R G_{X Y}^{f}(n)$.

Proof: Let $E\left(R G_{X Y}^{f}(n)\right)$ denote the set of edges of $R G_{X Y}^{f}(n)$. Note that

$$
\begin{aligned}
E\left(R G_{X Y}^{f}(n)\right)= & \left\{\left(\left(x^{n}, y^{n}\right),\left(x^{n}, y^{\prime n}\right)\right) \in S_{X^{n} Y^{n}} ; f\left(x_{i}, y_{i}\right) \neq f\left(x_{i}, y_{i}^{\prime}\right) \text { for some } i\right\} \\
& \cup\left\{\left(\left(x^{n}, y^{n}\right),\left(x^{\prime n}, y^{n}\right)\right) \in S_{X^{n} Y^{n}} ; f\left(x_{i}, y_{i}\right) \neq f\left(x_{i}^{\prime}, y_{i}\right) \text { for some } i\right\}
\end{aligned}
$$

Observe that each edge is of the form $\left(\left(x^{n}, y^{n}\right),\left(x^{n}, y^{\prime n}\right)\right)$ or $\left(\left(x^{n}, y^{n}\right),\left(x^{\prime n}, y^{n}\right)\right)$. We note that

(i) A can recover $f\left(X^{n}, Y^{n}\right)$ with zero-error $\Leftrightarrow$ for any $\left(x^{n}, y^{n}\right),\left(x^{n}, y^{\prime n}\right) \in S_{X^{n} Y^{n}}$ with $f\left(x_{i}, y_{i}\right) \neq f\left(x_{i}, y_{i}^{\prime}\right)$ for some $i, \phi_{C}\left(\phi_{A}\left(x^{n}\right), \phi_{B}\left(y^{n}\right)\right) \neq \phi_{C}\left(\phi_{A}\left(x^{n}\right), \phi_{B}\left(y^{\prime n}\right)\right)$.

(ii) B can recover $f\left(X^{n}, Y^{n}\right)$ with zero-error $\Leftrightarrow$ for any $\left(x^{n}, y^{n}\right),\left(x^{\prime n}, y^{n}\right) \in S_{X^{n} Y^{n}}$ with $f\left(x_{i}, y_{i}\right) \neq f\left(x_{i}^{\prime}, y_{i}\right)$ for some $i, \phi_{C}\left(\phi_{A}\left(x^{n}\right), \phi_{B}\left(y^{n}\right)\right) \neq \phi_{C}\left(\phi_{A}\left(x^{\prime n}\right), \phi_{B}\left(y^{n}\right)\right)$.

From (i) and (ii) above, it follows that $\mathrm{A}$ and $\mathrm{B}$ can recover $f\left(X^{n}, Y^{n}\right)$ with zero-error $\Leftrightarrow$ for any $\left(\left(x^{n}, y^{n}\right)\right.$, $\left.\left(x^{\prime n}, y^{\prime n}\right)\right) \in E\left(R G_{X Y}^{f}(n)\right), \phi_{C}\left(\phi_{A}\left(x^{n}\right), \phi_{B}\left(y^{n}\right)\right) \neq \phi_{C}\left(\phi_{A}\left(x^{\prime n}\right), \phi_{B}\left(y^{\prime n}\right)\right) \Leftrightarrow \phi_{C} \circ\left(\phi_{A} \times \phi_{B}\right)$ is a coloring of $R G_{X Y}^{f}(n)$. 
Lemma 3 For any $n \geq 1$, and given the encoding functions $\phi_{A}, \phi_{B}, \phi_{C}$, the nodes $A$ and $B$ can recover $f\left(X^{n}, Y^{n}\right)$ under the unrestricted input setup if and only if $\phi_{C} \circ\left(\phi_{A} \times \phi_{B}\right)$ is a coloring of $R G_{X Y}^{f,(u)}(n)$.

Proof: Let $E\left(R G_{X Y}^{f,(u)}(n)\right)$ denote the set of edges of $R G_{X Y}^{f,(u)}(n)$. Observe that

$$
\begin{aligned}
E\left(R G_{X Y}^{f,(u)}(n)\right)= & \left\{\left(\left(x^{n}, y^{n}\right),\left(x^{n}, y^{\prime n}\right)\right): \text { for some } i\left(\left(x_{i}, y_{i}\right),\left(x_{i}, y_{i}^{\prime}\right)\right) \in E\left(R G_{X Y}^{f,(u)}\right)\right\} \\
& \cup\left\{\left(\left(x^{n}, y^{n}\right),\left(x^{\prime n}, y^{n}\right)\right) \text { : for some } i\left(\left(x_{i}, y_{i}\right),\left(x_{i}^{\prime}, y_{i}\right)\right) \in E\left(R G_{X Y}^{f,(u)}\right)\right\} .
\end{aligned}
$$

We note that

(i) A can recover $f\left(X^{n}, Y^{n}\right)$ under the unrestricted input setup $\Leftrightarrow$ for any $\left(x^{n}, y^{n}\right),\left(x^{n}, y^{\prime n}\right)$ such that $f\left(x_{i}, y_{i}\right) \neq$ $f\left(x_{i}, y_{i}^{\prime}\right)$ for some $i$ where $\left(x_{i}, y_{i}\right),\left(x_{i}, y_{i}^{\prime}\right) \in S_{X Y}, \phi_{C}\left(\phi_{A}\left(x^{n}\right), \phi_{B}\left(y^{n}\right)\right) \neq \phi_{C}\left(\phi_{A}\left(x^{n}\right), \phi_{B}\left(y^{\prime n}\right)\right)$.

(ii) B can recover $f\left(X^{n}, Y^{n}\right)$ under the unrestricted input setup $\Leftrightarrow$ for any $\left(x^{n}, y^{n}\right),\left(x^{\prime n}, y^{n}\right)$ such that $f\left(x_{i}, y_{i}\right) \neq$ $f\left(x_{i}^{\prime}, y_{i}\right)$ for some $i$ where $\left(x_{i}, y_{i}\right),\left(x_{i}^{\prime}, y_{i}\right) \in S_{X Y}, \phi_{C}\left(\phi_{A}\left(x^{n}\right), \phi_{B}\left(y^{n}\right)\right) \neq \phi_{C}\left(\phi_{A}\left(x^{\prime n}\right), \phi_{B}\left(y^{n}\right)\right)$

From (i) and (ii) above, it follows that $\mathrm{A}$ and $\mathrm{B}$ can recover $f\left(X^{n}, Y^{n}\right)$ with zero-error $\Leftrightarrow$ for any $\left(\left(x^{n}, y^{n}\right)\right.$, $\left.\left(x^{\prime n}, y^{\prime n}\right)\right) \in E\left(R G_{X Y}^{f,(u)}(n)\right), \phi_{C}\left(\phi_{A}\left(x^{n}\right), \phi_{B}\left(y^{n}\right)\right) \neq \phi_{C}\left(\phi_{A}\left(x^{\prime n}\right), \phi_{B}\left(y^{\prime n}\right)\right) \Leftrightarrow \phi_{C} \circ\left(\phi_{A} \times \phi_{B}\right)$ is a coloring of $R G_{X Y}^{f,(u)}(n)$.

Proof of part (a): Lemma 2 implies that for encoding functions $\phi_{A}, \phi_{B}, \phi_{C}$ of any zero-error scheme, $\phi_{A}, \phi_{B}, \phi_{C} \circ$ $\left(\phi_{A} \times \phi_{B}\right)$ is a color cover for $R G_{X Y}^{f}(n)$. Similarly, for any color cover $\left(c_{A}, c_{B}, c_{C}\right)$ of $R G_{X Y}^{f}(n)$, let $\phi_{A}, \phi_{B}$ be any prefix-free encoding functions of $c_{A}$ and $c_{B}$ respectively. Since $c_{A} \times c_{B}$ is a refinement of $c_{C}$, there exists a mapping $\theta_{C}$ such that $c_{C}=\theta_{C} \circ\left(c_{A} \times c_{B}\right)$. Taking $\phi_{C}$ as any prefix-free encoding of $c_{C}$ yields a scheme with encoding functions $\left(\phi_{A}, \phi_{B}, \phi_{C}\right)$. Thus the result follows from the definition of the region $Z_{X, Y}^{f}$.

Proof of part (b) follows along the similar lines as that of part (a) using Lemma 3 .

\section{Proof of Theorem 3}

We first give some lemmas which are used to prove the theorem.

Lemma 4 (Covering Lemma, [32]). Let $(U, X, \hat{X}) \sim p(u, x, \hat{x})$ and $\epsilon^{\prime}<\epsilon$. Let $\left(U^{n}, X^{n}\right) \sim p\left(u^{n}, x^{n}\right)$ be a pair of random sequences with $\lim _{n \rightarrow \infty} P\left\{\left(U^{n}, X^{n}\right) \in T_{\epsilon^{\prime}}^{n}(U, X)\right\}=1$, and let $\hat{X}^{n}(m), m \in \mathcal{A}$, where $|\mathcal{A}| \geq 2^{n R}$, be random sequences, conditionally independent of each other and of $X^{n}$ given $U^{n}$, each distributed according to $\prod_{i=1}^{n} p_{\hat{X} \mid U}\left(\hat{x}_{i} \mid u_{i}\right)$. Then, there exists $\delta(\epsilon)$ that tends to zero as $\epsilon \rightarrow 0$ such that

$$
\lim _{n \rightarrow \infty} P\left\{\left(U^{n}, X^{n}, \hat{X}^{n}(m)\right) \notin T_{\epsilon}^{n} \text { for all } m \in \mathcal{A}\right\}=0
$$

if $R>I(X ; \hat{X} \mid U)+\delta(\epsilon)$. 
Lemma 5 (Markov Lemma, [32]). Suppose that $X \rightarrow Y \rightarrow Z$ form a Markov chain. Let $\left(x^{n}, y^{n}\right) \in T_{\epsilon^{\prime}}^{n}(X, Y)$, and $Z^{n} \sim p\left(z^{n} \mid y^{n}\right)$, where the conditional pmf $p\left(z^{n} \mid y^{n}\right)$ satisfies the following conditions:

1) $\lim _{n \rightarrow \infty} P\left\{\left(y^{n}, Z^{n}\right) \in T_{\epsilon^{\prime}}^{n}(Y, Z)\right\}=1$.

2) For every $z^{n} \in T_{\epsilon^{\prime}}^{n}\left(Z \mid y^{n}\right)$ and $n$ sufficiently large

$$
2^{-n\left(H(Z \mid Y)+\delta\left(\epsilon^{\prime}\right)\right)} \leq p\left(z^{n} \mid y^{n}\right) \leq 2^{-n\left(H(Z \mid Y)-\delta\left(\epsilon^{\prime}\right)\right)}
$$

for some $\delta\left(\epsilon^{\prime}\right)$ that tends to zero as $\epsilon^{\prime} \rightarrow 0$.

Then, for some sufficiently small $\epsilon^{\prime}<\epsilon$,

$$
\lim _{n \rightarrow \infty} P\left\{\left(x^{n}, y^{n}, Z^{n}\right) \in T_{\epsilon}^{n}(X, Y, Z)\right\}=1
$$

Lemma 6 [3 Lemma 4] There exists a function g such that $\forall(x, y) \in S_{X Y}, u_{2} \in \Gamma\left(G_{Y \mid X}^{f}\right)$ s.t. $y \in u_{2}, g\left(x, u_{2}\right)=$ $f(x, y)$, i.e., $f(x, y)$ can be computed from $u_{2}$ and $y$.

Lemma 7 There exists functions $g_{1}$ and $g_{2}$ such that for all $\left(x, y, u_{1}, u_{2}, w\right) \in \mathcal{X} \times \mathcal{Y} \times \Gamma\left(G_{X \mid Y}^{f}\right) \times \Gamma\left(G_{Y \mid X}^{f}\right) \times$ $\Gamma\left(\widetilde{R G}_{U_{1} U_{2}}^{f}\right)$ satisfying $\left(u_{1}, u_{2}\right) \in w$ and $p(x, y) p\left(u_{1} \mid x\right) p\left(u_{2} \mid y\right)>0, f(x, y)=g_{1}\left(x, u_{1}, w\right)=g_{2}\left(y, u_{2}, w\right)$.

Proof: For a given $X=x, U_{1}=u_{1}$ and $W=w$, let us consider the set of possible $y, A_{x, u_{1}, w}=\left\{y^{\prime}:\left(x, y^{\prime}\right) \in\right.$ $S_{X Y}$, and $p\left(u_{2}^{\prime} \mid y^{\prime}\right)>0$ for some $u_{2}^{\prime}$ s.t. $\left.\left(u_{1}, u_{2}^{\prime}\right) \in w\right\}$. Then we show that

Claim: $f\left(x, y^{\prime}\right)=f\left(x, y^{\prime \prime}\right) \forall y^{\prime}, y^{\prime \prime} \in A_{x, u_{1}, w}$.

Proof of the claim: Let us assume that for some $y^{\prime}, y^{\prime \prime} \in A_{x, u_{1}, w}, f\left(x, y^{\prime}\right) \neq f\left(x, y^{\prime \prime}\right)$. By definition of $A_{x, u_{1}, w}$, $\exists u_{2}^{\prime}, u_{2}^{\prime \prime} \in \Gamma\left(G_{Y \mid X}^{f}\right)$, such that $y^{\prime} \in u_{2}^{\prime}, y^{\prime \prime} \in u_{2}^{\prime \prime}$, and $\left(u_{1}, u_{2}^{\prime}\right),\left(u_{1}, u_{2}^{\prime \prime}\right) \in w$. But $\left(y^{\prime}, y^{\prime \prime}\right) \in E\left(G_{Y \mid X}^{f}\right)$, and so $y^{\prime \prime} \notin u_{2}^{\prime}$, and thus $u_{2}^{\prime} \neq u_{2}^{\prime \prime}$. From the conditions in the lemma and the definition of $A_{x, u_{1}, w}$, we have $p\left(x, u_{1}, y^{\prime}, u_{2}^{\prime}\right), p\left(x, u_{1}, y^{\prime \prime}, u_{2}^{\prime \prime}\right)>0$. Then by Definition 5. $\left(u_{1}, u_{2}^{\prime}\right)$ and $\left(u_{1}, u_{2}^{\prime \prime}\right)$ are connected in $\widetilde{R G_{U_{1} U_{2}}^{f}}$. This implies that $w$ is not an independent set of $\widetilde{R G}_{U_{1} U_{2}}^{f}$, which is a contradiction. This proves the claim.

Now, $g_{1}$ (resp. $g_{2}$ ) is defined as the unique function value $f(x, y)$ for all $y \in A_{x, u_{1}, w}$ (resp. $x \in A_{y, u_{2}, w}$ ).

We first give the proof of part (a).

Proof of part (a): In the following, we assume $\epsilon>\epsilon^{\prime}>\epsilon^{\prime \prime}>0$ and $|\mathcal{Q}|=1$. Let $\left\{U_{1}^{n}\left(m_{1}\right) \mid m_{1} \in\left\{1, \cdots, 2^{n R_{A}^{\prime}}\right\}\right\}$ be a set of independent sequences, each distributed according to $\prod_{i=1}^{n} p_{U_{1}}\left(u_{1 i}\right)$. Similarly, let $\left\{U_{2}^{n}\left(m_{2}\right) \mid m_{2} \in\right.$ $\left.\left\{1, \cdots, 2^{n R_{B}^{\prime}}\right\}\right\}$, be a set of independent sequences, each distributed according to $\prod_{i=1}^{n} p_{U_{2}}\left(u_{2 i}\right)$. Let $\left\{W^{n}\left(m_{3}\right) \mid m_{3} \in\right.$ $\left.\left\{1, \cdots, 2^{n R_{C}^{\prime}}\right\}\right\}$, be a set of independent sequences, each distributed according to $\prod_{i=1}^{n} p_{W}\left(w_{i}\right)$.

\section{Encoding at node A:}


For a given $x^{n}$, node A chooses an index $m_{1}$ (if any) such that $\left(x^{n}, U_{1}^{n}\left(m_{1}\right)\right) \in T_{\epsilon^{\prime \prime}}^{n}\left(X, U_{1}\right)$. The encoding at node $\mathrm{A}$ is given by

$$
\phi_{A}\left(x^{n}\right)= \begin{cases}m_{1} & \left(x^{n}, U_{1}^{n}\left(m_{1}\right)\right) \in T_{\epsilon^{\prime \prime}}^{n}\left(X, U_{1}\right) \\ x^{n} & \text { if }\left(x^{n}, U_{1}^{n}\left(m_{1}\right)\right) \notin T_{\epsilon^{\prime \prime}}^{n}\left(X, U_{1}\right) \forall m_{1} .\end{cases}
$$

By the covering lemma, if $R_{A}^{\prime}>I\left(X ; U_{1}\right)+\delta\left(\epsilon^{\prime \prime}\right)$ then

$$
\lim _{n \rightarrow \infty} \operatorname{Pr}\left(\exists m_{1},\left(X^{n}, U_{1}^{n}\left(m_{1}\right)\right) \in T_{\epsilon^{\prime \prime}}^{n}\left(X, U_{1}\right)\right)=1
$$

where $\delta\left(\epsilon^{\prime \prime}\right) \rightarrow 0$ as $\epsilon^{\prime \prime} \rightarrow 0$. Rate of the overall encoding is $R_{A}<R_{A}^{\prime}+\delta\left(\epsilon^{\prime \prime}\right)$ for large enough $n$ such that $\operatorname{Pr}\left(\forall m_{1},\left(X^{n}, U_{1}^{n}\left(m_{1}\right)\right) \notin T_{\epsilon^{\prime \prime}}^{n}\left(X, U_{1}\right)\right)<\delta\left(\epsilon^{\prime \prime}\right) / \log |\mathcal{X}|$. Thus, any rate $R_{A}>I\left(X ; U_{1}\right)+2 \delta\left(\epsilon^{\prime \prime}\right)$ is sufficient.

Encoding at node B is similar to that of the encoding at node A.

\section{Encoding at relay:}

If $R_{C}>I\left(W ; U_{1}, U_{2}\right)$, but $R_{C}<\max \left\{I\left(X ; U_{1}\right), I\left(Y ; U_{2}\right)\right\}$, the the relay uses the encoding as given in case 1 below. If $R_{C}>\max \left\{I\left(X ; U_{1}\right), I\left(Y ; U_{2}\right)\right\}$, then the relay uses the encoding as given in case 2 .

Case 1: $\max \left\{I\left(X ; U_{1}\right), I\left(Y ; U_{2}\right)\right\}>R_{C}>I\left(W ; U_{1}, U_{2}\right)$

The relay receives either an index $m_{1}$ or a $x^{n}$ sequence from node A. Similarly, from node B the relay receives $m_{2}$ or a $y^{n}$ sequence. If $m_{1}$ and $m_{2}$ are received, and $\left(u_{1}^{n}\left(m_{1}\right), u_{2}^{n}\left(m_{2}\right), w^{n}\left(m_{3}\right)\right) \in T_{\epsilon}^{n}\left(U_{1}, U_{2}, W\right)$ for some $m_{3}$, then any such $m_{3}$ is broadcasted by the relay. In any other case, the relay broadcasts both the received sequences. So the encoding at the relay is given by $\ddagger$

$$
\phi_{C}= \begin{cases}m_{3} \quad\left(u_{1}^{n}\left(m_{1}\right), u_{2}^{n}\left(m_{2}\right), w^{n}\left(m_{3}\right)\right) \in T_{\epsilon}^{n}\left(U_{1}, U_{2}, W\right) \\ \left(\phi_{A}\left(x^{n}\right), \phi_{B}\left(y^{n}\right)\right) & \text { otherwise. }\end{cases}
$$

Let $E_{n, \epsilon^{\prime}}$ be the event $\left(U_{1}^{n}\left(m_{1}\right), U_{2}^{n}\left(m_{2}\right)\right) \in T_{U_{1} U_{2}, \epsilon^{\prime}}^{n}$ at the relay. Then from the Markov lemma, we have $\lim _{n \rightarrow \infty} \operatorname{Pr}\left(E_{n, \epsilon^{\prime}}\right)=1$. By the covering lemma, if $R_{C}^{\prime}>I\left(W ; U_{1}, U_{2}\right)+\delta(\epsilon)$ then

$$
\lim _{n \rightarrow \infty} \operatorname{Pr}\left(\exists m_{3},\left(U_{1}^{n}\left(m_{1}\right), U_{2}^{n}\left(m_{2}\right), W^{n}\left(m_{3}\right)\right) \in T_{\epsilon}^{n}\left(U_{1}, U_{2}, W\right) \mid E_{n, \epsilon^{\prime}}\right)=1,
$$

where $\delta(\epsilon) \rightarrow 0$ as $\epsilon \rightarrow 0$. Rate of the overall encoding is $R_{C}<R_{C}^{\prime}+2 \delta(\epsilon)$ for large enough $n$ such that

$$
\operatorname{Pr}\left(E_{n, \epsilon^{\prime}} \cap \forall m_{3},\left(U_{1}^{n}\left(m_{1}\right), U_{2}^{n}\left(m_{2}\right), W^{n}\left(m_{3}\right)\right) \notin T_{\epsilon}^{n}\left(U_{1}, U_{2}, W\right)\right)<\delta(\epsilon) / \log \left(\mathcal{U}_{1} \cdot \mathcal{U}_{2}\right),
$$

and $\operatorname{Pr}\left(E_{n, \epsilon^{\prime}}^{c}\right)<\delta(\epsilon) / \log (a \cdot b)$, where $a=\max \left\{|\mathcal{X}|,\left|\mathcal{U}_{1}\right|\right\}$ and $b=\max \left\{|\mathcal{Y}|,\left|\mathcal{U}_{2}\right|\right\}$. Thus, any rate $R_{C}>$

\footnotetext{
$\dagger$ Transmission of an $x^{n}$ sequence is done by first converting the sequence to a binary sequence of maximum length $n$ log $|\mathcal{X}|$ bits. An extra prefix bit is added to distinguish the sequences $x^{n}$ and $m_{1}$. Similar operation is done for a $y^{n}$ sequence too.

$\ddagger$ This encoding can be represented by a prefix-free code using standard techniques, as outlined in the previous footnote.
} 
$I\left(W ; U_{1}, U_{2}\right)+3 \delta(\epsilon)$ is sufficient.

Case 2: $R_{C}>\max \left\{I\left(X ; U_{1}\right), I\left(Y ; U_{2}\right)\right\}$

Let us consider the case where the relay receives $m_{1}$ and $m_{2}$ from node $\mathrm{A}$ and $\mathrm{B}$ respectively, such that $\left(u_{1}^{n}\left(m_{1}\right), u_{2}^{n}\left(m_{2}\right)\right) \in T_{U_{1} U_{2}, \epsilon^{\prime}}^{n}$. Then the relay broadcasts the XOR of the binary representations of $m_{1}$ and $m_{2}$ (after padding zeros to the shorter sequence). In any other case, as in scheme 1, the relay broadcasts both the received sequences. So the encoding at the relay is given by $\$$

$$
\phi_{C}= \begin{cases}m_{1} \oplus m_{2} \quad\left(u_{1}^{n}\left(m_{1}\right), u_{2}^{n}\left(m_{2}\right)\right) \in T_{\epsilon^{\prime}}^{n}\left(U_{1}, U_{2}\right) \\ \left(\phi_{A}\left(x^{n}\right), \phi_{B}\left(y^{n}\right)\right) \quad \text { otherwise. }\end{cases}
$$

By using the Markov lemma as before, rate of the overall encoding is $R_{C}<\max \left\{R_{A}, R_{B}\right\}+2 \delta\left(\epsilon^{\prime}\right)$ for large enough $n$ such that $\operatorname{Pr}\left(\left(U_{1}^{n}\left(m_{1}\right), U_{2}^{n}\left(m_{2}\right)\right) \notin T_{U_{1} U_{2}, \epsilon^{\prime}}^{n}\right)<\delta\left(\epsilon^{\prime}\right) / \log \left(\left|\mathcal{U}_{1}\right| .\left|\mathcal{U}_{2}\right|\right)$. Thus any rate $R_{C}>\max \left\{R_{A}, R_{B}\right\}+2 \delta\left(\epsilon^{\prime}\right)$ is sufficient.

\section{Decoding at node A:}

If the relay follows the encoding scheme given in case 1, then node A performs the decoding procedure given in case 1 below. Othersiwe, it follows the decoding operation given in case 2.

\section{Case 1:}

Node A receives either $m_{3}, m_{2}$ or $y^{n}$. We show that node A computes $f\left(x_{i}, y_{i}\right)$ with zero-error $\forall i$ for which $\left(x_{i}, y_{i}\right) \in S_{X Y}$. Let us consider a pair $\left(x^{n}, y^{n}\right)$ such that $\left(x_{i}, y_{i}\right) \in S_{X Y}$ for some $i$.

\section{Subcase 1: Node A receives $m_{3}$}

In this case, node $\mathrm{A}$ and node $\mathrm{B}$ had chosen $m_{1}$ and $m_{2}$ such that $\left(x^{n}, u_{1}^{n}\left(m_{1}\right)\right) \in T_{\epsilon^{\prime \prime}}^{n}\left(X, U_{1}\right)$ and $\left(y^{n}, u_{2}^{n}\left(m_{2}\right)\right) \in$ $T_{\epsilon^{\prime \prime}}^{n}\left(Y, U_{2}\right)$ respectively, and at the relay $\left(u_{1}^{n}\left(m_{1}\right), u_{2}^{n}\left(m_{2}\right), w^{n}\left(m_{3}\right)\right) \in T_{\epsilon}^{n}\left(U_{1}, U_{2}, W\right)$.

As a robustly typical sequence can not have a zero-probability component (see (1)), the sequence $u_{1}^{n}\left(m_{1}\right)$ chosen by node A satisfies $p\left(u_{1 i} \mid x_{i}\right)>0 \forall i$, since $\left(x^{n}, u_{1}^{n}\left(m_{1}\right)\right) \in T_{\epsilon^{\prime \prime}}^{n}\left(X, U_{1}\right)$. Similarly, the sequence $u_{2}^{n}\left(m_{2}\right)$ chosen by node B satisfies $p\left(u_{2 i} \mid y_{i}\right)>0 \forall i$, and the sequence $w^{n}\left(m_{3}\right)$ chosen by the relay satisfies $p\left(w_{i} \mid u_{1 i}, u_{2 i}\right)>0 \forall i$. Hence $p\left(u_{1 i} \mid x_{i}\right) p\left(u_{2 i} \mid y_{i}\right) p\left(w_{i} \mid u_{1 i}, u_{2 i}\right)>0$. Thus by Lemma 7, if $p\left(x_{i}, y_{i}\right)>0$ then node A can compute $f\left(x_{i}, y_{i}\right)$ from $x_{i}, u_{1 i}$ and $w_{i}$ with zero-error.

Subcase 2: Node A receives $m_{2}$

In this case node $\mathrm{B}$ had $m_{2}$ such that $\left(y^{n}, u_{2}^{n}\left(m_{2}\right)\right) \in T_{\epsilon^{\prime \prime}}^{n}\left(Y, U_{2}\right)$. This shows that $p\left(u_{2 i}\left(m_{2}\right) \mid y_{i}\right)>0$ for all $i$. Thus by Lemma 6, node A can recover $f\left(x_{i}, y_{i}\right)$ with zero-error for all $i$ such that $p\left(x_{i}, y_{i}\right)>0$.

Subcase 3: Node A receives $y^{n}$

In this case node A can compute $f\left(x_{i}, y_{i}\right)$ for all $i$.

\footnotetext{
$\S$ This encoding can be represented by a prefix-free code using standard techniques, as outlined in the footnote in page 23
} 


\section{Case 2:}

Node A receives either $m_{1} \oplus m_{2}, m_{2}$ or $y^{n}$. Let us first consider the case where node A receives $m_{1} \oplus m_{2}$. Since node A has $m_{1}$, it can decode $m_{2}$ from $m_{1} \oplus m_{2}$ by XORing the received message with $m_{1}$. Zero-error function computation at node A from $m_{2}$ and $x^{n}$ follows from Lemma 6. Decoding for all other cases is the same as decoding in scheme 1.

Node B follows the similar decoding procedure as that of node A. Now using time sharing random variable $Q$ gives the achievability of every triple $\left(R_{A}, R_{B}, R_{C}\right)$ in $\mathcal{R}_{I}$ for some $p(q) p\left(w \mid u_{1}, u_{2}, q\right) p\left(u_{1} \mid x, q\right) p\left(u_{2} \mid y, q\right)$. This completes the proof of part (a).

Proof of part (b): For the function computation problem in Example 1, we show that $\exists\left(U_{1}^{\prime}, U_{2}^{\prime}\right.$, $\left.W^{\prime}\right)$ s.t. $I\left(W^{\prime} ; U_{1}^{\prime}, U_{2}^{\prime}\right)=\log 2$, and $\max \left\{I\left(X ; U_{1}\right), I\left(Y ; U_{2}\right)\right\}>\log 2$ for any choice of $\left(U_{1}, U_{2}\right)$. In this example, graphs $G_{X \mid Y}^{f}$ and $G_{Y \mid X}^{f}$ are pentagon graphs.The complementary graph entropy of a pentagon graph with uniform distribution is shown to be $\frac{1}{2} \log 5[11]$. Since the graph entropy is greater than or equal to the complementary graph entropy, we get $\max \left\{I\left(X ; U_{1}\right), I\left(Y ; U_{2}\right)\right\}>\frac{1}{2} \log 5$ for any choice of $\left(U_{1}, U_{2}\right)$. Let us consider a scheme for the choice of $U_{1}=\{X\}$ and $U_{2}=\{Y\}$. Then $R_{A}=R_{B}=\log 5$. For this choice of $\left(U_{1}, U_{2}\right)$, the graph $\widetilde{R G}_{U_{1} U_{2}}^{f}$ is same as the graph $R G_{X Y}^{f}$ which is shown in Fig. 3a. Let us choose

$$
W=\left\{\begin{array}{l}
\left\{\left(u_{1}, u_{2}\right) \mid u_{1}=u_{2}\right\} \text { if } U_{1}=U_{2} \\
\left\{\left(u_{1}, u_{2}\right) \mid u_{1} \neq u_{2}\right\} \text { if } U_{1} \neq U_{2} .
\end{array}\right.
$$

Then $W$ is a binary random variable with uniform distribution and satisfies all the conditions in Theorem 3 . Here, since $W$ is a function of $\left(U_{1}, U_{2}\right)$, we get $I\left(W ; U_{1}, U_{2}\right)=H(W)=\log 2$, and we have $\max \left\{I\left(X ; U_{1}\right), I\left(Y ; U_{2}\right)\right\}>$ $\log 2$. Then we have the desired result.

For the function computation problem in Example 2, we show that $\exists\left(U_{1}^{\prime}, U_{2}^{\prime}\right)$ s.t. $\max \left\{I\left(X ; U_{1}^{\prime}\right), I\left(Y ; U_{2}^{\prime}\right)\right\}=\frac{2}{3}$, and $I\left(W ; U_{1}, U_{2}\right)>\frac{2}{3}$ for any $\left(U_{1}, U_{2}, W\right)$. To prove this, we consider the same of choices of $U_{1}$ and $U_{2}$ given in Section III-A and we use the following claim.

Claim 1 The only conditional distribution $p_{U_{1} \mid X}$ achieving $R_{A}=\frac{2}{3}$ for the function computation problem in Example 2 is $p_{U_{1} \mid X}(a \mid 2)=p_{U_{1} \mid X}(b \mid 2)=\frac{1}{2}$.

Proof: To prove the above claim, we need to show that $I\left(X ; U_{1}\right)$ is strictly convex in $p_{U_{1} \mid X}$. Let us take $p_{U_{1} \mid X}(a \mid 2)=p$, for $0<p<1$. Then $I\left(X ; U_{1}\right)$ is a function of $p$ which can be written as

$$
I\left(X ; U_{1}\right)=f(p)=-\frac{1}{3}(1+p) \log \frac{1}{3}(1+p)-\frac{1}{3}(2-p) \log \frac{1}{3}(2-p)+\frac{1}{3} p \log p+\frac{1}{3}(1-p) \log (1-p) .
$$


Next we show that $f^{\prime \prime}(p)>0$, for $0<p<1$.

$$
\begin{aligned}
f^{\prime}(p) & =\frac{1}{3} \log \frac{1}{3}(1+p)-\frac{1}{3} \log \frac{1}{3}(2-p)+\frac{1}{3} \log p-\frac{1}{3} \log (1+p) . \\
f^{\prime \prime}(p) & =\frac{1}{1+p}+\frac{1}{2-p}+\frac{1}{3} \frac{1}{p}+\frac{1}{3} \frac{1}{1-p} .
\end{aligned}
$$

Then we have $f^{\prime \prime}(p)>0, \quad$ for $0<p<1$. This proves the claim.

For Example 2, the confusability graphs $G_{X \mid Y}^{f}$ and $G_{Y \mid X}^{f}$ are the same and it is shown in Fig. 4a. For uniform distribution on its vertices, the graph entropy of the graph shown in Fig. $4 \mathrm{a}$, is computed as $\frac{2}{3}$ in Example 1 in [3]. So we have $H_{G_{X \mid Y}^{f}}(X)=H_{G_{Y \mid X}^{f}}(Y)=\frac{2}{3}$. Then we get $\left(\frac{2}{3}, \frac{2}{3}, \frac{2}{3}\right) \in \mathcal{R}_{I 2}$. Claim 11 shows that we have to choose $p_{U_{1} \mid X}(a \mid 2)=p_{U_{1} \mid X}(b \mid 2)=p_{U_{2} \mid Y}(c \mid 2)=p_{U_{2} \mid Y}(d \mid 2)=\frac{1}{2}$ to achieve the rates $R_{A}=R_{B}=\frac{2}{3}$. For this choice of $\left(U_{1}, U_{2}\right)$, let us compute the joint distribution of $\left(U_{1}, U_{2}\right)$. Note that $\left(U_{1}, U_{2}\right)=(a, c)$ has non zero joint probability with $(X, Y)$ when either $(X, Y)=(1,2)$ or $(X, Y)=(2,1)$. By marginalizing over $(X, Y)$, we get $p_{U_{1}, U_{2}}(a, c)=\frac{1}{6}$. Similarly, we get $p_{U_{1}, U_{2}}(b, d)=\frac{1}{6}, p_{U_{1}, U_{2}}(a, d)=p_{U_{1}, U_{2}}(b, c)=\frac{1}{3}$.

As we have seen before the graph $\widetilde{R G}_{U_{1} U_{2}}^{f}$ is a "square" graph which is shown in Fig. $4 \mathrm{c}$. The minimum $R_{C}$ achievable by Scheme 1 in this case is $H_{G_{U_{1} U_{2}}^{f}}\left(U_{1}, U_{2}\right)$. For this graph $\widetilde{R G}_{U_{1} U_{2}}^{f}$, the only two maximal independent sets are $\{(a, c),(b, d)\}$ and $\{(a, d),(b, c)\}$. Let $W$ be a random variable distributed over $\{\{(a, c),(b, d)\},\{(a, d),(b, c)\}\}$. Since each node of the graph $\widetilde{R G}_{U_{1} U_{2}}^{f}$ is contained in only one of the maximal independent set, we have $w$ as a function of $\left(u_{1}, u_{2}\right)$. Then $R_{C}=I\left(W ; U_{1}, U_{2}\right)=H(W)=H\left(\frac{1}{3}\right) \approx 0.91$. This shows that for the above choice of $\left(U_{1}, U_{2}\right)$, the minimum $R_{C}$ achievable using scheme 1 is $H\left(\frac{1}{3}\right)$. Since $H\left(\frac{1}{3}\right)>\frac{2}{3}$, we get $\left(\frac{2}{3}, \frac{2}{3}, \frac{2}{3}\right) \notin \mathcal{R}_{I 1}$. This completes the proof of part (b).

\section{Proof of Theorem 4}

Since $p(x, y)>0 \forall(x, y) \in \mathcal{X} \times \mathcal{Y}, p\left(x^{n}, y^{n}\right)>0$ for any $x^{n} \in \mathcal{X}^{n}$ and $y^{n} \in \mathcal{Y}^{n}$. Let $f^{n}\left(x^{n}, y^{n}\right)$ denote $\left(f\left(x_{1}, y_{1}\right), \cdots, f\left(x_{n}, y_{n}\right)\right)$. If the relay node cannot compute the function with zero-error, it implies that there exists $x^{n}, y^{n}, x^{\prime n}, y^{\prime n}$ such that $\phi_{A}\left(x^{n}\right)=\phi_{A}\left(x^{\prime n}\right), \phi_{B}\left(y^{n}\right)=\phi_{B}\left(y^{\prime n}\right)$ and $f^{n}\left(x^{n}, y^{n}\right) \neq f^{n}\left(x^{\prime n}, y^{\prime n}\right)$. We either have $f^{n}\left(x^{n}, y^{n}\right) \neq f^{n}\left(x^{n}, y^{\prime n}\right)$ or $f^{n}\left(x^{n}, y^{\prime n}\right) \neq f^{n}\left(x^{\prime n}, y^{\prime n}\right)$. W.1.o.g., let us assume that $f^{n}\left(x^{n}, y^{n}\right) \neq f^{n}\left(x^{n}, y^{\prime n}\right)$. For both pairs $\left(x^{n}, y^{n}\right)$ and $\left(x^{n}, y^{\prime n}\right)$, node A receives $\phi_{C}\left(\phi_{A}\left(x^{n}\right), \phi_{B}\left(y^{n}\right)\right)$ from the relay. Then node A cannot compute the function since the relay's message and $X^{n}$ are the same for these pairs, but the values of the function are different. So we get a contradiction. This proves the result.

\section{V. $\epsilon$-ERror COMPUTATION: Proofs of Theorems 5-6}

Proof of Lemma (1), part (a): Let us consider the cut between node A and the super-node consisting of B and C. Then it is the function computation problem with side information considered in [3] where the decoder with side 
information $Y$ wants to compute a function $f(X, Y)$. They showed that the optimal $\epsilon$-error rate for this problem is $H_{G_{X \mid Y}^{f}}(X \mid Y)$. This implies that $R_{A} \geq H_{G_{X \mid Y}^{f}}(X \mid Y)$. Similarly, $R_{B} \geq H_{G_{Y \mid X}^{f}}(Y \mid X)$. The lower bound for $R_{C}$ follows from the cut set bound by considering the cut $(\{C\},\{A, B\})$ and assuming that the relay knows $(X, Y)$.

Proof of part $(b)$ : Let us consider a scheme where nodes $\mathrm{A}$ and $\mathrm{B}$ encode $X^{n}$ and $Y^{n}$ to messages $m_{1}$ and $m_{2}$ by the scheme given by Orlitsky and Roche in [3], and the relay broadcasts both these messages. From the result of [3], $R_{A}=H_{G_{X \mid Y}^{f}}(X \mid Y), R_{B}=H_{G_{Y \mid X}^{f}}(Y \mid X)$, and $R_{C}=H_{G_{X \mid Y}^{f}}(X \mid Y)+H_{G_{Y \mid X}^{f}}(Y \mid X)$ are achievable using this scheme. Now let us consider another scheme where $X$ and $Y$ are communicated to the relay from $\mathrm{A}$ and $\mathrm{B}$. Then the relay first computes $f(X, Y)$ and then uses Slepian-Wolf binning to compress it at a rate $R_{C}=\max \{H(Z \mid X), H(Z \mid Y)\}$. Then nodes A and B can compute $f(X, Y)$ with negligible probability of error. The rates $R_{A}=H(X), R_{B}=H(Y)$, and $R_{C}=\max \{H(Z \mid X), H(Z \mid Y)\}$ are achievable for this scheme.

\section{A. Proof of Theorem 5}

Proof of part $(a):$ The scheme used to prove the achievability of $\mathcal{R}_{I 1}^{\epsilon}$ is similar to that of $\mathcal{R}_{I}$ in Theorem 3 . Nodes A and B follow Berger-Tung coding scheme [33]. (We refer the reader to Theorem 12.1 in [32].) At node A, like in scheme 1 in Theorem 3 , a codebook $\left\{U_{1}^{n}\left(m_{1}\right) \mid m_{1} \in\left\{1, \cdots, 2^{n R_{A}^{\prime}}\right\}\right\}$ is used. The codebook is randomly binned into $2^{n R_{A}}$ bins. If a $u_{1}^{n}\left(m_{1}\right)$ is found which is jointly typical with $x^{n}$, then its bin index $b_{1}$ is sent. If no such $u_{1}^{n}$ is found in the codebook, then a randomly chosen bin index is sent. Node B encodes in a similar way.

The relay can correctly recover $\left(m_{1}, m_{2}\right)$ from $b_{1}$ and $b_{2}$ with high probability if $R_{A}>I\left(X ; U_{1} \mid U_{2}\right), R_{B}>$ $I\left(Y ; U_{2} \mid U_{1}\right)$ and $R_{A}+R_{B}>I\left(X, Y ; U_{1}, U_{2}\right)$. Let the reconstructed messages be $\left(\hat{m}_{1}, \hat{m}_{2}\right)$. The relay follows Wyner-Ziv coding scheme where a codebook $\left\{W^{n}\left(m_{3}\right) \mid m_{3} \in\left\{1, \cdots, 2^{n R_{C}^{\prime}}\right\}\right\}$ is randomly binned into $2^{n R_{C}}$ bins. If the relay finds a $w^{n}\left(m_{3}\right)$ which is jointly typical with $\left(u_{1}^{n}\left(\hat{m}_{1}\right), u_{2}^{n}\left(\hat{m}_{2}\right)\right)$, then it broadcasts the bin index of $w^{n}\left(m_{3}\right)$. Otherwise a randomly chosen bin index is broadcasted. Node A can decode $m_{3}$ correctly with high probability if

$$
\begin{aligned}
R_{C} & \stackrel{(a)}{=} I\left(W ; U_{1}, U_{2}\right)-I\left(W ; X, U_{1}\right)+\epsilon \\
& =H\left(W \mid X, U_{1}\right)-H\left(W \mid U_{1}, U_{2}\right)+\epsilon \\
& \stackrel{(b)}{=} H\left(W \mid X, U_{1}\right)-H\left(W \mid U_{1}, U_{2}, X\right)+\epsilon \\
& =I\left(W ; U_{2} \mid X, U_{1}\right)+\epsilon .
\end{aligned}
$$

Here in $(a)$, we have taken the size of the bin as $2^{n\left(I\left(W ; X, U_{1}\right)+\epsilon^{\prime}\right)}$, and $(b)$ follows from the Markov chain $W-$ $U_{1} U_{2}-X$. Similarly node B can decode $m_{3}$ with high probability if $R_{C} \geq I\left(W ; U_{1} \mid Y, U_{2}\right)$.

Let the reconstructed messages at nodes $\mathrm{A}$ and $\mathrm{B}$ be $\hat{m}_{3}^{A}$ and $\hat{m}_{3}^{B}$ respectively. Then $w^{n}\left(\hat{m}_{3}^{A}\right)$ will be jointly typical with $\left(x^{n}, u_{1}^{n}\left(m_{1}\right), y^{n}, u_{2}^{n}\left(m_{2}\right)\right)$ with high probability. For such a $w^{n}\left(\hat{m}_{3}^{A}\right)$, for all $i$ such that $p\left(x_{i}, y_{i}\right)>0$, 
we get $p\left(u_{1 i} \mid x_{i}\right) p\left(u_{2 i} \mid y_{i}\right) p\left(w_{i} \mid u_{1 i}, u_{2 i}\right)>0$ using robust typicality. Thus by Lemma 7 , node A can compute $f\left(x_{i}, y_{i}\right)$ from $x_{i}, u_{1 i}$ and $w_{i}$. Node $\mathrm{B}$ computes the function in a similar way.

Now let us consider the encoding schemes used to obtain the rate region $\mathcal{R}_{I 2}^{\epsilon}$. Node A encodes $X^{n}$ to an index $m_{1}$ using the scheme given by Orlitsky and Roche in [3]. Using the same scheme, node B encodes $Y^{n}$ to an index $m_{2}$ with rate $R_{B}$. Once the relay receives both the messages, it broadcasts the XOR of the binary representation of $m_{1}$ and $m_{2}$ (after appending zeros to the shorter sequence). Nodes A recovers message $m_{2}$ from $\left(m_{1}, m_{1} \oplus m_{2}\right)$. Then node $\mathbf{A}$ follows the decoding operation given in [3] to compute the function. Similar decoding operation is performed at node B. By the result of [3], $R_{A}=H_{G_{X \mid Y}^{f}}(X \mid Y), R_{B}=H_{G_{Y \mid X}^{f}}(Y \mid X)$, and $R_{C}=\max \left\{H_{G_{X \mid Y}^{f}}(X \mid Y), H_{G_{Y \mid X}^{f}}(Y \mid X)\right\}$ are achievable using this scheme.

Proof of part $(b)$ : Let us consider computing $X \cdot Y$ (AND function) for $\operatorname{DSBS}(p)(X, Y)$. Here both the confusability graphs $G_{X \mid Y}^{f}$ and $G_{Y \mid X}^{f}$ are complete. This implies $H_{G_{X \mid Y}^{f}}(X \mid Y)=H(X \mid Y)$ and $H_{G_{Y \mid X}^{f}}(Y \mid X)=H(Y \mid X)$. Since $H(X \mid Y)=H(Y \mid X)=H(p)$, we get $\mathcal{R}_{I 2}^{\epsilon}=\left\{\left(R_{A}, R_{B}, R_{C}\right): R_{A}, R_{B}, R_{C} \geq H(p)\right\}$. Now let us consider the achievable scheme of $\mathcal{R}_{I 1}^{\epsilon}$ in Theorem 5 for this example. Since both the confusability graphs are complete, the only choice for $U_{1}$ and $U_{2}$ are $U_{1}=\{X\}$ and $U_{2}=\{Y\}$. For this choice of $U_{1}$ and $U_{2}$, the relay can recover $X$ and $Y$ by Berger-Tung coding scheme. Then the relay can compute the function $Z=f(X, Y)$. For a given $Z=z$, let us consider the set of all $(x, y), A_{z}=\left\{(x, y): f(x, y)=z\right.$, and $\left.(x, y) \in S_{X Y}\right\}$. Let us choose $W=A_{Z}$. Then we get $R_{C}=\max \{H(Z \mid X), H(Z \mid Y)\}=\frac{1}{2} H(p)$, which is the minimum possible $R_{C}$ by Lemma 1 . So we get $\mathcal{R}_{I 1}^{\epsilon}$ as

$$
\left\{\left(R_{A}, R_{B}, R_{C}\right): R_{A} \geq H(p), R_{B} \geq H(p), R_{A}+R_{B} \geq 1+H(p), R_{C} \geq \frac{1}{2} H(p)\right\} .
$$

Then we have $(H(p), H(p), H(p)) \in \mathcal{R}_{I 2}^{\epsilon} \backslash \mathcal{R}_{I 1}^{\epsilon}$ and $\left(1, H(p), \frac{1}{2} H(p)\right) \in \mathcal{R}_{I 1}^{\epsilon} \backslash \mathcal{R}_{I 2}^{\epsilon}$.

\section{B. Proof of Theorem 6}

We use the following lemma to prove Theorem 6 For $f_{1}, f_{2}$ of $(X, Y)$, let the random variables $Z_{1}$ and $Z_{2}$ denote $f_{1}(X, Y)$ and $f_{2}(X, Y)$ respectively.

Lemma 8 If $E\left(R G_{X Y}^{f_{1}}\right) \subseteq E\left(R G_{X Y}^{f_{2}}\right)$, then $H\left(Z_{1} \mid Z_{2}, X\right)=0$ and $H\left(Z_{1} \mid Z_{2}, Y\right)=0$.

Proof: We prove that if $E\left(R G_{X Y}^{f_{1}}\right) \subseteq E\left(R G_{X Y}^{f_{2}}\right)$, then $H\left(Z_{1} \mid Z_{2}, X\right)=0$. The other case follows similarly. For a given $X=x$ and $Z_{2}=z_{2}$, let us consider the set of all $y, A_{x z_{2}}=\left\{y^{\prime}: f_{2}\left(x, y^{\prime}\right)=z_{2}\right.$ and $\left.\left(x, y^{\prime}\right) \in S_{X Y}\right\}$. Then by the definition of $R G_{X Y}^{f_{2}}, f_{2}\left(x, y^{\prime}\right)=f_{2}\left(x, y^{\prime \prime}\right) \forall y^{\prime}, y^{\prime \prime} \in A_{x z_{2}}$. Further, since $E\left(R G_{X Y}^{f_{1}}\right) \subseteq E\left(R G_{X Y}^{f_{2}}\right)$, $f_{1}\left(x, y^{\prime}\right)=f_{1}\left(x, y^{\prime \prime}\right)$. Let us denote this unique value by $z_{1}:=f_{1}\left(x, y^{\prime}\right)$. Then we have $\operatorname{Pr}\left\{Z_{1}=z_{1} \mid X=x, Z_{2}=\right.$ $\left.z_{2}\right\}=1$ and $H\left(Z_{1} \mid Z_{2}, X\right)=0$. 
Proof of part a): Lemma 8 shows that if $E\left(R G_{X Y}^{f_{1}}\right) \subseteq E\left(R G_{X Y}^{f_{2}}\right)$, then $Z_{1}$ is a function of $\left(Z_{2}, X\right)$ as well as a function of $\left(Z_{2}, Y\right)$. This implies that if node A can recover $Z_{2}^{n}$ from $M_{C}$ and $X^{n}$ with some probability of error, then it can compute $Z_{1}^{n}$ with at most the same probability of error. Similar arguments hold for computing $Z_{1}^{n}$ at node B. This shows that $\mathcal{R}_{(0)}^{R N}\left(f_{1}, X, Y\right) \supseteq \mathcal{R}_{(0)}^{R N}\left(f_{2}, X, Y\right)$ and $\mathcal{R}_{(\epsilon)}^{R N}\left(f_{1}, X, Y\right) \supseteq \mathcal{R}_{(\epsilon)}^{R N}\left(f_{2}, X, Y\right)$.

Part (b) follows from part (a).

\section{CONCLUSION}

In this work, we studied the function computation problem in a bidirectional relay network (Fig. 11). Function computation problem has been addressed from an information theoretic point of view for unidirectional networks before, e.g. [1]-[3], [15]. To the best of our knowledge, this is the first work which addressed the function computation problem for a bidirectional network from an information theoretic point of view. We considered our function computation problem on this network for correlated sources under zero-error and $\epsilon$-error criteria and proposed single-letter inner and outer bounds for achievable rates. We studied the function computation problem in a broadcast network (Fig. 2), where we showed that the optimal broadcast rate is the same under zero-error and $\epsilon$-error criteria.

\section{APPENDIX A}

\section{SOURCE CODING UNDER ZERO-ERROR VS. $\epsilon$-ERROR}

We now mention a result which connects zero-error with $\epsilon$-error. We believe this result is folklore. We provide it here for completeness. The result in the following lemma can be extended/applied to our source coding network.

Lemma 9 Let us consider a source coding problem with side information where the encoder knows $X$ and the decoder has the side information $Y$ and wants to recover $X$. If there is a zero-error prefix free code of rate $R$, then for any $\delta>0$, the rate $R+\delta$ is achievable under $\epsilon$-error.

Proof: Consider a zero-error prefix free code of length $n$ and rate $R$. Let $M_{A}$ denote the encoded message. Since the average length of any prefix free encoding is lower bounded by the entropy of the source, we get $n R \geq H\left(M_{A}\right)$. Now let us consider a block encoding of $N$ messages $M_{A}$ under $\epsilon$-error. For any $\delta>0$, there exists an $N$ such that by random binning of $M_{A}^{N}$ symbols at a rate $H\left(M_{A}\right)+\delta$, the decoder can reconstruct $M_{A}$ with arbitrarily small probability of error. Since the source vectors $X^{n}$ can be reconstructed with zero-error from $M_{A}$, the decoder can decode $X$ with arbitrarily small probability of error. This proves the lemma. 


\section{REFERENCES}

[1] J. Körner, and K. Marton, "How to encode the modulo-two sum of binary sources," IEEE Transactions on Information Theory, vol. 25, no. 2, pp. 219-221, Mar. 1979.

[2] T. Han and K. Kobayashi, "A dichotomy of functions $F(X, Y)$ of correlated sources $(X, Y)$ from the viewpoint of the achievable rate region," IEEE Transactions on Information Theory, vol. 33, no. 1, pp. 69-76, Jan. 1987.

[3] A. Orlitsky and J. R. Roche, “Coding for computing,” IEEE Transactions on Information Theory, vol. 47, no. 3, pp. 903-917, Mar. 2001.

[4] H. Kowshik and P. R. Kumar, "Optimal function computation in directed and undirected graphs," IEEE Transactions on Information Theory, vol. 58, no. 6, pp. 3407-3418, Jun. 2012.

[5] B. K. Rai and B. K. Dey, “On network coding for sum-networks,” IEEE Transactions on Information Theory, vol. 58, no. 1, pp. 50-63, Jan. 2012.

[6] V. Shah, B. K. Dey and D. Manjunath, "Network flows for function computation," IEEE Journal on Selected Areas in Communications, vol. 31, no. 4, pp. 714-730, Apr. 2013.

[7] A. D. Wyner, J. K. Wolf and F. M. J. Willems, “Communicating via a processing broadcast satellite," IEEE Transactions on Information Theory, vol. 48, no. 6, pp. 1243-1249, Jun. 2002.

[8] H. Su, and A. El Gamal, “Two-way source coding through a relay," IEEE International Symposium on Information Theory, Jun. 2010.

[9] L. H. Witsenhausen, "The zero-error side information problem and chromatic numbers," IEEE Transactions on Information Theory, vol. 22, no. 5, pp. 592-593, Jan. 1976.

[10] N. Alon and A. Orlitsky, "Source coding and graph entropies," IEEE Transactions on Information Theory, vol. 42, no. 5, pp. 1329-1339, Sept. 1996.

[11] P. Koulgi, E. Tuncel, S. L. Regunathan, and K. Rose, “On zero-error source coding with decoder side information," IEEE Transactions on Information Theory, vol. 49, no. 1, pp. 99-111, Jan. 2003.

[12] J. Körner and G. Longo, “Two-step encoding of finite memoryless sources," IEEE Transactions on Information Theory, vol. 19, no. 6, pp. 778-782, Nov. 1973.

[13] D. Slepian and J. K. Wolf, "Noiseless coding of correlated information sources," IEEE Transactions on Information Theory, vol. 19, no. 4, pp. 471-480, Jul. 1973.

[14] P. Koulgi, E. Tuncel, S. L. Regunathan, and K. Rose, “On zero-error coding of correlated sources," IEEE Transactions on Information Theory, vol. 49, no. 11, pp. 2856-2873, Nov. 2003.

[15] O. Shayevitz, "Distributed computing and the graph entropy region," IEEE Transactions on Information Theory, vol. 60, no. 6, pp. 3435-3449, Jun. 2014.

[16] N. Alon, E. Lubetzky, U. Stav, A. Weinstein, and A. Hassidim, "Broadcasting with side information," in 49th Ann. IEEE Symp. Found. Comput. Sci., Philadelphia, PA, Oct. 2008, pp. 823-832.

[17] E. Tuncel, "Slepian-Wolf coding over broadcast channels," IEEE Transactions on Information Theory, vol. 52, no. 4, pp. 1469-1482, Apr. 2006.

[18] Y. Wu, "Broadcasting when receivers know some messages a priori," in Proc. IEEE International Symposium onInformation Theory, Nice, France, Jun. 2007.

[19] G. Kramer and S. Shamai, "Capacity for classes of broadcast channels with receiver side information," in Proc. IEEE Information Theory Workshop, California, USA, Sep. 2007.

[20] A. Kimura, T. Uyematsu, and S. Kuzuoka, "Universal coding for correlated sources with complementary delivery," IEICE Transactions Fundamentals, vol. E90-A, no. 9, pp. 1840-1847, Sep. 2007. 
[21] R. Timo, A. Grant, and G. Kramer, "Lossy broadcasting with complementary side information," IEEE Trans. Inf. Theory, vol. 59, no. 1, pp. 104-131, Jan. 2013.

[22] Z. Bar-Yossef, Y. Birk, T. S. Jayram, and T. Kol, "Index coding with side information," IEEE Trans. Inf. Theory, vol. 57, no. 3, pp. 1479-1494, Mar. 2011.

[23] M. Effros, S. El Rouayheb, and M. Langberg, “An Equivalence Between Network Coding and Index Coding” IEEE Trans. Inf. Theory, vol. 61, no. 5, pp. 2478-2487, May. 2015.

[24] F. Arbabjolfaei and Y. H. Kim, "Structural properties of index coding capacity using fractional graph theory," in Proc. IEEE International Symposium onInformation Theory, Hong Kong, Jun. 2015.

[25] H. Maleki, V. R. Cadambe, and S. A. Jafar, "Index coding an interference alignment perspective," IEEE Trans. Inf. Theory, vol. 60, no. 9, pp. 54025432, Sep. 2014.

[26] M. Langberg and M. Effros, "Network coding: Is zero error always possible?" in Proc. 49th Ann. Allerton Conf. Comm. Control Comput., Monticello, IL, Sep. 2011, pp. 1478-1485.

[27] J. Körner, "Coding of an information source having ambiguous alphabet and the entropy of graphs," in Proc. 6th Prague Conf Inf. Theory, 1973, pp. 411-425.

[28] G. Simonyi, “Graph entropy: A survey,” in Proc. DIMACS, vol. 20, 1995, pp. 399-441.

[29] J. Körner, "Fredman-Komlós bounds and information theory," SIAM J. Algebraic and Discrete Methods, vol. 7, no. 4, pp. 560-570, Oct. 1986.

[30] E. Tuncel, J. Nayak, P. Koulgi, and K. Rose, “On Complementary Graph Entropy,” IEEE Transactions on Information Theory, vol. 55, no. 6, pp. 2537-2546, Jun. 2009.

[31] H. H. Permuter, Y. Steinberg, and T. Weissman, "Two-way source coding with a helper," IEEE Transactions on Information Theory, vol. 56, no. 6, pp. 2905-2919, Jun. 2010.

[32] A. El Gamal and Y. H. Kim, Network Information Theory, Canbridge, U.K, Cambridge Univ. Press, 2011.

[33] T. Berger, "Multiterminal source coding," in G. Longo, editor, The Information Theory Approach to Communications, pp. 171-231, Springer-Verlag, New York, 1977. 